ARTICLE

\title{
Identification of novel DNA-damage tolerance genes reveals regulation of translesion DNA synthesis by nucleophosmin
}

Omer Ziv ${ }^{1}$, Amit Zeisel ${ }^{2}$, Nataly Mirlas-Neisberg ${ }^{1}$, Umakanta Swain $^{1}$, Reinat Nevo ${ }^{1}$, Nir Ben-Chetrit ${ }^{3}$, Maria Paola Martelli ${ }^{4}$, Roberta Rossi ${ }^{4}$, Stefan Schiesser ${ }^{5}$, Christine E. Canmann ${ }^{6}$, Thomas Carell ${ }^{5}$, Nicholas E. Geacintov ${ }^{7}$, Brunangelo Falini ${ }^{4}$, Eytan Domany ${ }^{2} \&$ Zvi Livneh ${ }^{1}$

Cells cope with replication-blocking lesions via translesion DNA synthesis (TLS). TLS is carried out by low-fidelity DNA polymerases that replicate across lesions, thereby preventing genome instability at the cost of increased point mutations. Here we perform a twostage siRNA-based functional screen for mammalian TLS genes and identify 17 validated TLS genes. One of the genes, NPM1, is frequently mutated in acute myeloid leukaemia (AML). We show that NPM1 (nucleophosmin) regulates TLS via interaction with the catalytic core of DNA polymerase- $\eta$ (pol $\eta$ ), and that NPM1 deficiency causes a TLS defect due to proteasomal degradation of pol $\eta$. Moreover, the prevalent NPM1c + mutation that causes NPM1 mislocalization in $\sim 30 \%$ of AML patients results in excessive degradation of poln. These results establish the role of NPM1 as a key TLS regulator, and suggest a mechanism for the better prognosis of AML patients carrying mutations in NPM1.

\footnotetext{
${ }^{1}$ Department of Biological Chemistry, Weizmann Institute of Science, Rehovot 76100, Israel. ${ }^{2}$ Department of Physics of Complex Systems, Weizmann Institute of Science, Rehovot 76100, Israel. ${ }^{3}$ Department of Biological Regulation, Weizmann Institute of Science, Rehovot 76100, Israel. ${ }^{4}$ Institute of Hematology, University of Perugia, Perugia 06100, Italy. ${ }^{5}$ Department of Chemistry, Ludwig Maximilians University Munich, Munich 81377, Germany. ${ }^{6}$ Department of Pharmacology, University of Michigan, Ann Arbor, Michigan 48109, USA. ${ }^{7}$ Department of Chemistry, New York University, New York, New York 10003, USA. Correspondence and requests for materials should be addressed to Z.L. (email: zvi.livneh@weizmann.ac.il).
} 
T he high abundance of DNA damage has led to the evolution of a variety of DNA-damage tolerance and repair pathways that minimize potential deleterious effects on DNA replication and gene expression, thereby preventing genome instability and a plethora of pathological conditions ${ }^{1}$. Most of the damage is repaired by accurate DNA repair mechanisms, primarily during the G1 phase of the cell cycle. However, during S phase, DNA replication encounters DNA lesions that have escaped repair, or that were newly formed, leading to the arrest of replication forks and/or the formation of single-stranded gaps, which may further lead to the formation of double-stranded breaks (DSB) and genome instability ${ }^{2}$. These replication obstacles are dealt with by DNAdamage tolerance ${ }^{3}$, of which two main strategies are known: (1) translesion DNA synthesis (TLS), whereby specialized low-fidelity DNA polymerases replicate across the damaged DNA region in a process that is inherently error prone $e^{4-7}$; and (2) homologydependent repair in which the gap opposite the DNA lesion is filled-in by either physical transfer of the complementary strand from the sister chromatid or by using the latter as a template for copying the missing strand (also termed error-free post-replication repair or template switch repair $)^{8-13}$. The importance of TLS is highlighted by the hereditary disease xeroderma pigmentosum variant (XPV), which is characterized by sunlight sensitivity and very high predisposition to skin cancer, caused by germline mutations that inactivate DNA polymerase- $\eta$ (pol $\eta$ ), a major TLS DNA polymerase ${ }^{14,15}$. The realization that TLS maintains a low mutagenic burden despite its inherent error-prone nature, and protects cells against genome instability and cancer, raised great interest in this process ${ }^{5,6}$. TLS usually involves two DNA polymerases: an inserter, which incorporates a nucleotide opposite the damaged template base, and an extender, which continues DNA synthesis beyond the damaged base ${ }^{16,17}$. Several layers of TLS regulation are known, including damaged-induced monoubiquitination of proliferating cell nuclear antigen (PCNA), the sliding DNA clamp, which serves to recruit TLS DNA polymerases to damaged sites in the DNA ${ }^{18-24}$ and clearance of TLS polymerases from the DNA by the activity of DVC1 and p97 (refs 21,22). In terms of cell physiology, TLS largely operates uncoupled from DNA replication, during late $S$ and early G2 phases of the cell cycle ${ }^{25-27}$, and is also regulated by the DNAdamage response via the ataxia telangiectasia and Rad3-related (ATR) protein ${ }^{28,29}$ and via p53/p21 (refs 30-32). The high complexity of TLS in mammalian cells and its involvement in the development of cancer drug resistance ${ }^{33-37}$ highlights the importance of understanding how this process is regulated. While previous studies aimed to systematically identify TLS genes in Saccharomyces cerevisiae proved to be highly useful in the field, to the best of our knowledge, screening for mammalian TLS genes has not been yet reported, and high-throughput assays for mammalian TLS are currently not available.

Here we present the development of a high-throughput assay for TLS in mammalian cells, and its implementation in screening 1,000 candidate genes. We further describe the validation of 17 novel TLS players, and the mechanistic and clinical insights revealed by investigating one of them, nucleophosmin, encoded by the NPM1 gene. We show that NPM1 regulates TLS by protecting poln from proteasomal degradation, and that a deficiency in NPM1 as well as expression of the acute myeloid leukaemia (AML)-related NPM1c + mutation results in decreased pol $\eta$ levels and defective TLS. Our results uncover multiple novel TLS regulators in mammalian cells and implicate NPM1 in the proteolytic regulation of TLS polymerases.

\section{Results}

Two-stage functional siRNA screen for mammalian TLS genes. We performed a two-stage functional short interfering RNA
(siRNA) screen designed to identify new mammalian TLS genes. In the first stage, we assayed ultraviolet sensitivity using an XPA cell line that is deficient in nucleotide excision repair (NER), and therefore defective in the repair of ultraviolet-induced DNA damage. Consequently, ultraviolet survival of the XPA cells exhibits a greater dependence on DNA-damage tolerance compared with NER-proficient cells ${ }^{38}$, making the screen more selective to DNA-damage tolerance genes. siRNAs that were identified in this stage as significantly affecting ultraviolet survival were re-screened with a second more stringent assay, which measured TLS. This strategy was used to screen 1,000 siRNAs directed to genes involved in DNA repair, ubiquitination and deubiquitination, cell cycle regulation and cancer. The ultravioletsensitivity screen (Fig. 1a) was performed in three biological replicas, exhibiting good reproducibility (Fig. 1b). Of the 1,000 genes assayed, we found 192 genes for which knockdown resulted in elevated ultraviolet sensitivity, and 45 genes that reduced ultraviolet sensitivity (false discovery rate (FDR) $<8 \%$; Fig. 1c, Supplementary Data 1, Supplementary Table 1 and Supplementary Fig. 1). Known TLS genes, as well as genes related to the ATR DNA-damage response pathway, but not other DNA repair pathways, were highly represented among the hits (Fig. 1d), suggesting that the XPA deficiency indeed enriched for genes involved in TLS.

The second screening stage was performed using a newly developed high-throughput TLS assay. It is a modification of an assay based on plasmids containing a site-specific DNA lesion opposite a gap, previously successfully used to study TLS $17,32,38$. In the new assay, the gap lesion is positioned between a cytomegalovirus (CMV) promoter and a firefly luciferase (Fluc) reporter gene (Fig. 2a). The presence of a gap in the transcribed strand does not allow expression of the Fluc gene, unless the missing segment of the coding strand is synthesized by TLS. A lesion-free gapped plasmid expressing Renilla luciferase (Rluc) served to normalize for transfection and gap-filling efficiencies (Fig. 2b). Of note, the product of a successful TLS event in the Fluc system still carries the lesion on the non-transcribed strand, which might interfere with Fluc expression. To test this possibility, we constructed the expected TLS product, namely a fully double-stranded plasmid with the lesion on the nontranscribed strand, and found that its Fluc expression in XPA cells was essentially identical to that obtained with a control plasmid without the lesion (Supplementary Fig. 2a).

The high-throughput TLS system was utilized to screen 237 siRNA hits from the primary screen for involvement in TLS across each of two major ultraviolet irradiation-induced DNA lesions, namely thymine-thymine 6-4 photoproduct (TT 6-4 PP) and thymine-thymine cyclobutane pyrimidine dimer (TT CPD; Fig. 2c-e). Screening was performed in four independent biological replicas performed on different days and exhibited high reproducibility (Fig. 2f,g). To normalize for differential damage-independent knockdown effects on Fluc and Rluc expression, each siRNA was also tested using Fluc and Rluc plasmids both carrying gaps but no lesions (NLs), and the results served to correct the data (Supplementary Fig. 2b,c). TLS elevation or inhibition scores were calculated with respect to control siRNAs, and were subjected to statistical analysis (Supplementary Fig. 2d-f). This screen resulted in 47 novel hits and 12 known TLS genes (FDR $<10 \%$; Supplementary Data 1 and Supplementary Table 1). Importantly, known TLS genes, but not other DNA repair pathways, were further enriched among the hits (Fig. $2 \mathrm{~h}$ and Supplementary Table 2), indicating high specificity of the screening method towards the TLS pathway. The ATR signalling pathway was also enriched to some extent, in agreement with recent reports ${ }^{28,29}$. Repetitive examination of each hit using four different siRNA oligos, to minimize off-target 

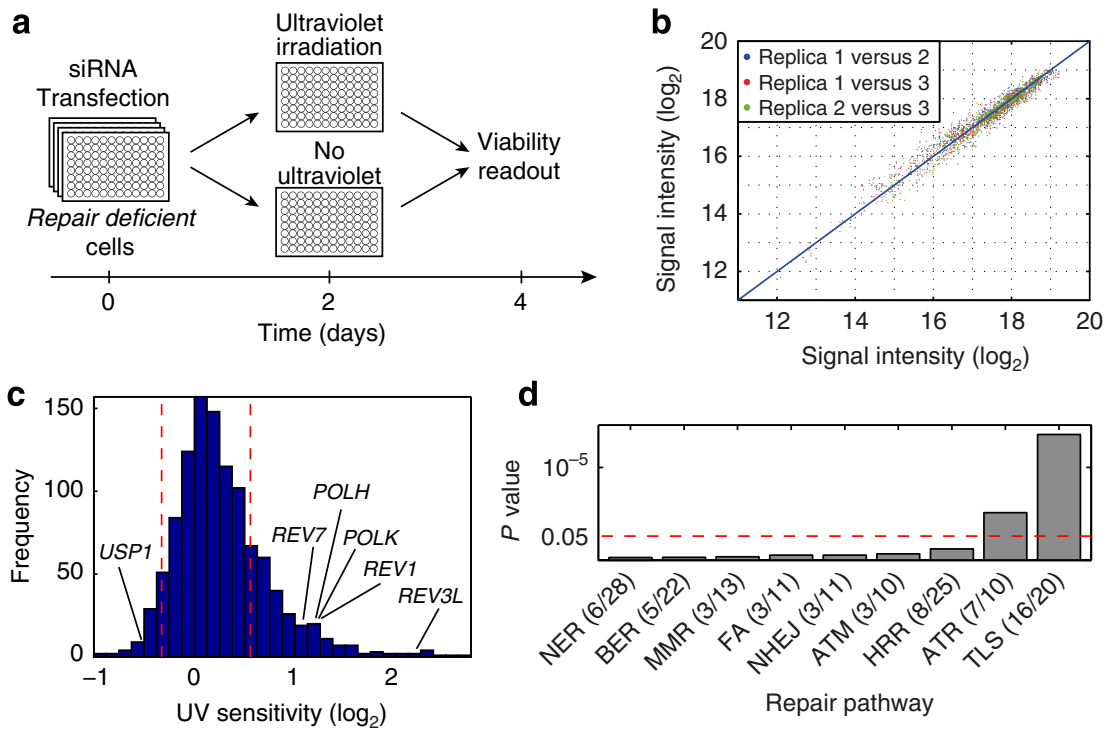

Figure 1 | Primary screen for ultraviolet sensitivity in NER-deficient human cells. (a) An outline of the primary screen. In brief, NER-deficient XPA cells were transfected with the siRNA libraries on day 0 , exposed to ultraviolet $\mathrm{C}$ (UVC) irradiation $\left(1 \mathrm{~J} \mathrm{~m}^{-2}\right.$ ) on day 2 and analysed for cell viability on day 4 . (b) Data reproducibility. Luminescence values of three biological replicas that were preformed on different days were plotted against each other. (c) Histogram describing ultraviolet-sensitivity fold change (FC) caused by the siRNAs (median values over the three replicas). Dashed red lines denote the distribution borders of negative control samples. (d) Enrichment of TLS, DNA repair and DNA-damage response pathways within the screen hits. Dashed red line corresponds to a hypergeometric test $P$ value 0.05 . NER, nucleotide excision repair; BER, base excision repair; MMR, mismatch repair; FA, the Fanconi anemia pathway; NHEJ, non-homologous end joining; HRR, homologous recombination repair; ATM and ATR, the two main DNA-damage response pathways. See also Supplementary Fig. 1, Supplementary Data 1 and Supplementary Table 1.

effects, excluded 30 of the genes, yielding 17 validated novel TLS genes (Table 1).

Functional validation of candidate TLS genes. To further validate the hits, a second version of the TLS assay was developed based on quantitative PCR (qPCR) readouts rather than gene expression (Fig. 3a). In brief, following TLS in mammalian cells, the plasmids were extracted under alkaline conditions, which denatured gapped plasmids but not the covalently closed products of TLS or the control gap-filling reactions. Remnants of gapped plasmids were digested with S1 single-stranded endonuclease. TLS efficiency was calculated as the ratio between the products of qPCRs that targeted the filled-in gap-lesion plasmid and the filled-in control gapped plasmid, and was normalized to control siRNA-treated samples (Fig. 3a). The qPCR-based TLS assay was utilized to examine the involvement of 10 selected hits in TLS across the TT 6-4 PP ultraviolet lesion in mouse embryonic fibroblasts (MEFs). In parallel, TLS was measured across the major tobacco smoke-induced DNA adduct benzo $[a]$ pyrene-guanine (BP-G), a non-ultraviolet lesion. Six hits, namely Papd7, Ruvbl2, Trip11, Npm1, Abh2 and Ube2e1, significantly affected TLS across both DNA lesions, although the effects obtained for Abh2 and Ube2e1 were rather small (Fig. 3b, $t$-test, $P$ values $<0.05$; knockdown efficiencies are shown in Fig. 3c). Cyld, Mcm3, Dclre1a and Ercc4 affected TLS across TT 64 PP but not across BP-G (Fig. 3b, Cyld knockdown reduced TLS across BP-G, but gave a marginal $P$ value of 0.06 ). Of notice, all the 10 tested human genes were validated in the qPCR-based TLS assay with at least one DNA lesion in MEFs. Four genes were further tested for their impact on TLS extent and mutagenicity in a gapped plasmid assay in which the readout is based on transformation of Escherichia coli cells, and therefore enables determining the sequence signature of TLS events ${ }^{17,32,38}$. Knocking down the expression of each of the tested genes, namely Cyld, Npm1, Papd7 and Ruvbl2, significantly reduced TLS efficiency across the BP-G adduct (Fig. 3d; two-tailed $t$-test, $P$ values $<0.02$ ). DNA sequence analysis showed that in control cells TLS was $47 \%$ accurate (insertion of a dCMP opposite BP-G), and 53\% were point mutations, most of which (87\%) were caused by insertion of dAMP opposite the lesion (Fig. 3e), consistent with previous results ${ }^{17,39}$. Interestingly, knocking down the expression of each of the four tested genes also caused, in addition to the decrease in the extent of TLS, a lower error rate among the TLS events (Fig. 3e).

NPM1 regulates DNA poln promoted TLS. Among the novel TLS genes, we concentrated for further analysis on NPM1, a gene frequently mutated in $\mathrm{AML}^{40}$. NPM1 (also termed nucleophosmin or B23) is a multi-functional protein involved in diverse cellular processes such as ribosome biogenesis, histone assembly, protein chaperoning and cell proliferation ${ }^{41}$. To explore the possibility that NPM1 affects poln-promoted TLS, we tested TLS across three lesions that are bypassed by poln: the sunlight-induced TT CPD and two adducts formed by the chemotherapy drug cisplatin, namely cisPt-GG and cisPt-GTG. As can be seen in Fig. 4a, in MEF cells in which Npm1 was knocked down, TLS across each of the three lesions was significantly reduced (two-tailed $t$-test, $P$ values $<0.02$ ), with the TT CPD being most affected. In contrast, knocking down Npm1 in pol $\eta$-deficient $\mathrm{PolH}^{-/-}$MEF cells did not significantly affect TLS across the TT CPD lesion (Supplementary Fig. 3a,b), further suggesting that the effect of NPM1 on TLS across TT CPD is mediated via pol $\eta$. This effect was not indirectly mediated via APE1, the base excision repair endonuclease that is regulated by NPM1, since knocking down the expression of Ape1 in the MEF cells had no effect on TLS across TT CPD (Supplementary Fig. 3c,d). Since NPM1 was reported to control the levels of PCNA $^{42}$, we examined whether the effect on TLS is via reducing PCNA monoubiquitination, which is needed for effective recruitment of pol $\eta$ to damaged sites in $\mathrm{DNA}^{19,20}$ and for 


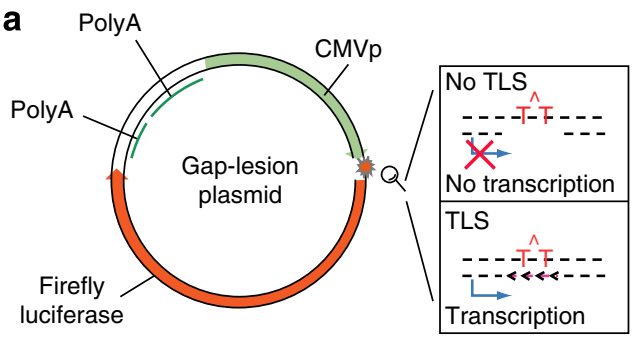

C

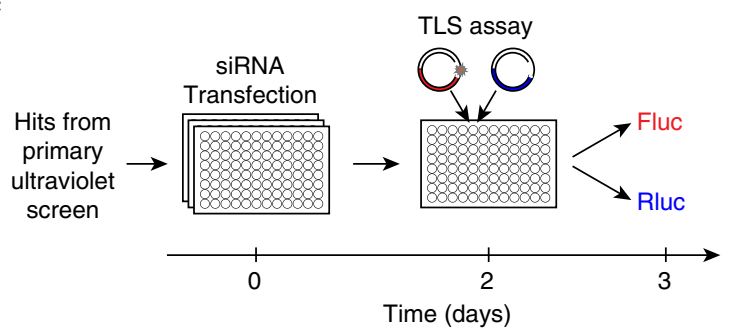

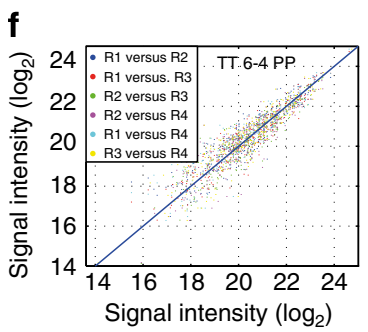

b
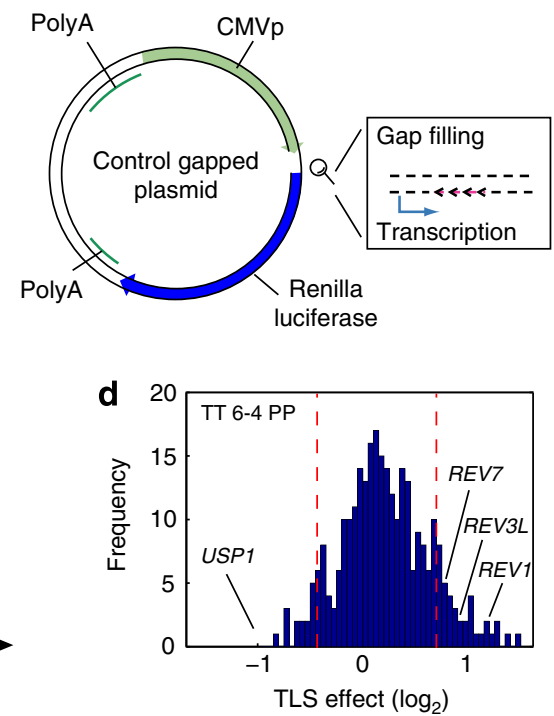
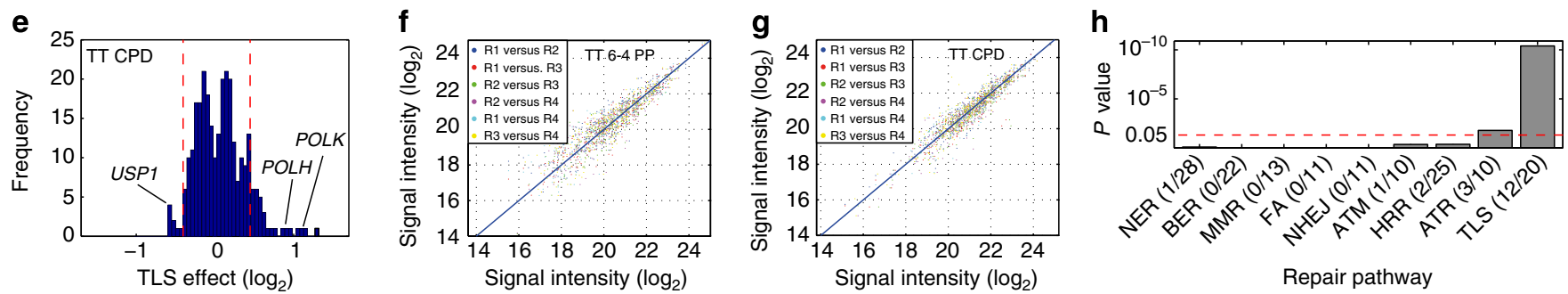

Figure 2 | Secondary functional TLS screen. $(\mathbf{a}, \mathbf{b})$ The plasmids used for the secondary TLS screen: A Fluc-expressing gapped plasmid carrying a site-specific DNA lesion on the non-transcribed strand and a gap on the transcribed strand (a) and a control Rluc-expressing gapped plasmid without a lesion (b). (c) An outline of the secondary TLS screen. In brief, XPA cells were transfected with the siRNA on day 0 and with the reporter plasmids on day 2 . Fluc and Rluc signals were measured on day 3. (d,e) Histograms describing TLS FC effects caused by the siRNAs (median values over four replicas that were preformed on different days). Dashed red lines denote the distribution borders of negative control samples. Data from the TT 6-4 PP TLS screen is presented in d, and that from the TT CPD TLS screen in $\mathbf{e . ~}(\mathbf{f}, \mathbf{g})$ Luminescence values of the four biological replicas plotted against each other from the TT 6-4 PP screen (f), and the TT CPD screen (g). (h) Enrichment of TLS, DNA repair and DNA-damage response pathways within the screen hits. The dashed red line corresponds to a hypergeometric test $P$ value 0.05. See also Supplementary Fig. 2, Supplementary Data 1 and Supplementary Tables 1 and 2.

effective TLS $^{23}$. Interestingly knocking down the expression of NPM1 increased PCNA monoubiquitination rather than decreasing it (Supplementary Fig. 4). This rules out the possibility that the TLS deficiency is owing to a PCNAubiquitination defect. A possible explanation for this observation is that the TLS defect caused by knockdown of NPM1 increases the number of stalled replication forks that leads to increased PCNA monoubiquitination.

NPM1 interacts with DNA poln in the nucleus. We next examined the interaction between pol $\eta$ and NPM1 by coimmunoprecipitation (co-IP) of the endogenous NPM1 with endogenous pol $\eta$. As can be seen in Fig. 4b, immunoprecipitation (IP) of poln with a specific antibody caused co-precipitation of NPM1. No NPM1 was observed when a control IgG was used (Fig. 4b, lane 2). To probe the subcellular compartment in which NPM1 and poln interact, we resorted to the proximity ligation assay (PLA). As can be seen in Fig. 4c, interaction foci between poln and NPM1 were clearly observed in human MRC5sv cells and localized mainly in the nucleus (the green spots in Fig. 4c upper panel, and quantified in Fig. 4d). These interaction foci were not observed in control $X P V$ cells that lack poln (Fig. 4c middle panel, and Fig. 4d), but appeared in XPV cells complemented with the POLH gene, encoding poln (Fig. 4c lower panel, and Fig. 4d). The interaction foci appear to be distributed throughout the nucleus but were excluded from the nucleoli (Supplementary Fig. 5a). Similarly, in MRC5sv cells co-expressing green fluorescent protein (GFP)-tagged poln and mCherry-tagged NPM1, GFP-poln was distributed throughout the nucleus and in reduced amounts in the nucleoli (Supplementary Fig. 5b). Taken together, these results indicate that NPM1 interact with pol $\eta$ in the nucleoplasm.

We next examined whether DNA-damage induction affects the interaction between NPM1 and poln. Exposure of the MRC5sv cells to ultraviolet irradiation resulted in a decrease in the amount of pol $\eta$ while the total levels of NPM1 were not significantly altered (Fig. 4e, input blot). Interestingly, following ultraviolet irradiation the interaction between pol $\eta$ and NPM1 was transiently lost, and reappeared at later time points, as detected by co-IP (Fig. 4e). Similarly, analysis by the PLA showed a transient loss of interaction between poln and NPM1 following ultraviolet irradiation (Fig. 4f, quantified in Fig. 4g). Ultraviolet irradiation of MRC5sv cells co-expressing GFP-tagged pol $\eta$ and mCherry-tagged NPM1 resulted in recruitment of poln but not of NPM1 into replication foci (Supplementary Fig. 5b), further supporting the observation that NPM1 releases poln following ultraviolet irradiation. To identify the region of poln that interacts with NPM1, a series of FLAG-poln deletion mutants was constructed and expressed in HEK293 cells. While IP of 
Table 1 | Summary of the novel TLS genes identified by the 2-stage screen.

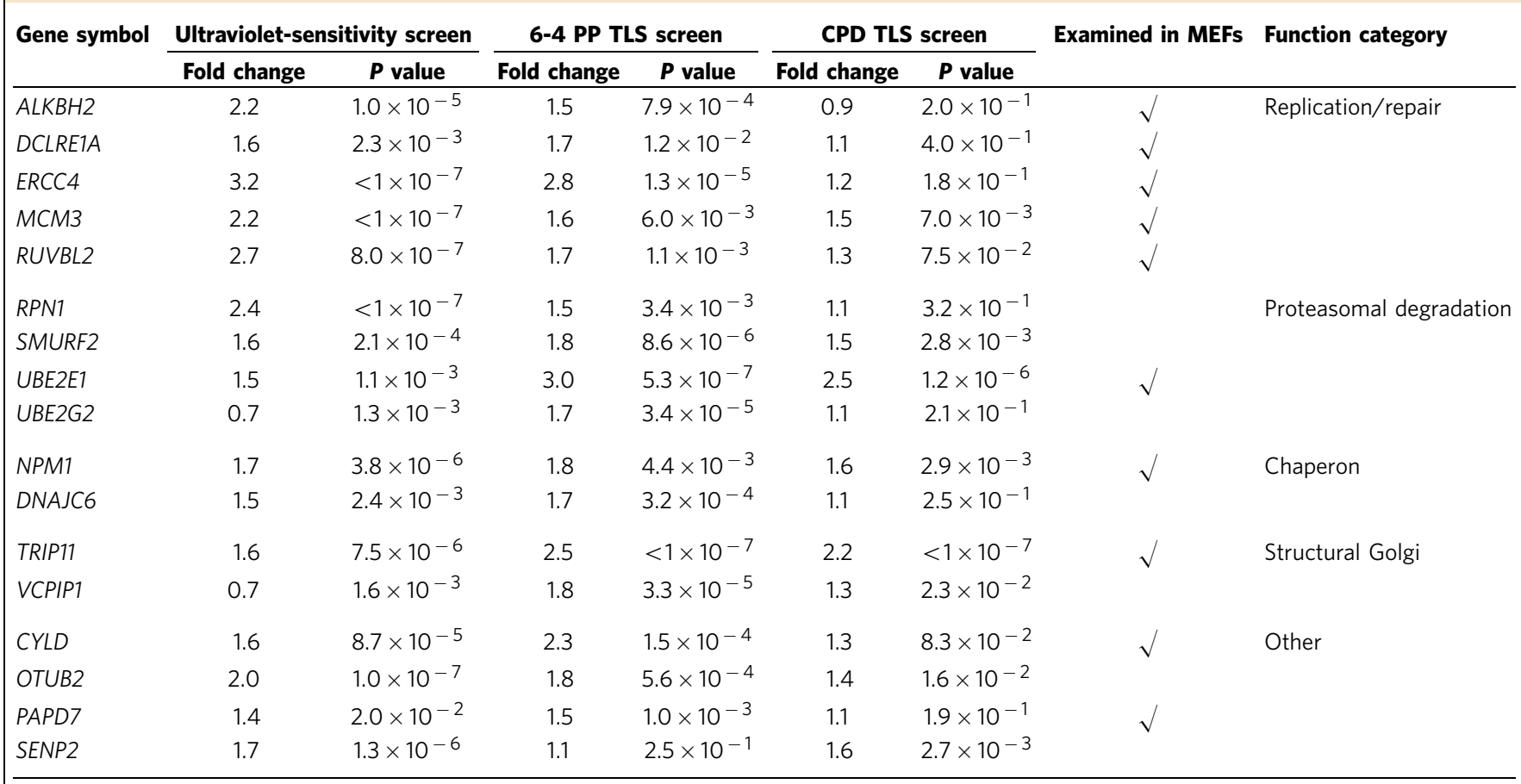

The table lists the 17 novel TLS genes identified by the two-stage screen, divided into functional categories.

NPM1 resulted in co-precipitation of FLAG-poln mutants lacking its regulatory carboxy terminus (Fig. $4 \mathrm{~h}$ lanes $2-7$, and Fig. 4i), it failed to co-precipitate the FLAG-poln mutants lacking the catalytic core of the polymerase (Fig. 4h lanes 8-9, and Fig. 4i). This suggests that NPM1 interacts with the catalytic core of poln.

NPM1 protects DNA pol $\eta$ against proteasomal degradation. To examine whether NPM1 affects poln stability, we measured by flow cytometry the level of ectopically expressed GFP-POLH in MRC5sv cells that stably express this construct. Knocking down NPM1 in these cells resulted in a threefold decrease in the expression of the GFP-pol $\eta$ construct (Fig. 5a, median values). When cells expressing GFP-RAD18, a gene fusion of the main E3 ligase responsible for monoubiquitination of PCNA were analysed, no reduction in RAD18 expression was obtained following knockdown of NPM1 (Supplementary Fig. 6), which is in agreement with the functional PCNA ubiquitination in these cells. Next, we tested whether NPM1 regulates the protein level of endogenous poln. Knocking down the expression of NPM1 in $X P A$ cells caused a significant fourfold decrease in the amount of the NPM1 protein (Fig. 5b, lanes 1 and 2). A similar result was obtained with the MRC5sv cells (3.4-fold decrease; Fig. 5c, lanes 1 and 2). Under these conditions, the amount of poln was significantly reduced both in the XPA cells (3.8-fold decrease; Fig. 5b, lanes 1 and 2) and the repair-proficient MRC5sv cells (2.9-fold decrease, Fig. 5c, lanes 1 and 2), with no significant change in POLH mRNA level, as measured by qPCR (Fig. 5d). A similar effect was observed in ultraviolet-irradiated cells (Fig. 5b lanes 3 and 4, and Fig. 5c lanes 3 and 4, corresponding to XPA and MRC5sv cells, respectively). Importantly, ectopic expression of an siRNA-resistant NPM1 construct rescued the reduction in poln caused by the silencing of endogenous NPM1 (Fig. 5e). Thus, a reduction in the amount of NPM1 causes a decrease in the amount of endogenous pol $\eta$ at the post-transcriptional level. A similar reduction was also observed with polk, which is another
TLS DNA polymerase, but not with polt, the closest homologue of poln in mammalian cells (Fig. 5f). Inhibiting the proteasome using MG132 caused an increase in poln levels in cells pre-treated with siRNA against NPM1, but not in those treated with control siRNA (Fig. 5g). This indicates that NPM1 has an important function in maintaining the stability of pol $\eta$, and protecting it from proteasomal degradation. The E3 ubiquitin ligases PIRH2 and MDM2 were implicated in the proteasomal degradation of poln. Nevertheless, knocking down each of these enzymes or both, in addition to knocking down NPM1, did not rescue the pol $\eta$ level (Supplementary Fig. 7a,b), suggesting that the degradation of pol $\eta$ upon NPM1 deficiency is not mediated solely by PIRH2 or by MDM2.

NPM1c + mutations phenocopy the deficiency in DNA poln. The C terminus of NPM1 is mutated in $\sim 30 \%$ of all AML patients, resulting in loss of the nucleolar localization signal and the generation of a de novo nuclear export signal. This leads to mislocalization of mutant NPM1 (designated NPM1c +), and partially of wild-type (wt) NPM1 interacting with it, in the cytoplasm $^{40,43}$. These mutations strongly correlate with better response of patients to chemotherapy and better clinical outcome $e^{44,45}$. Because of the role of NPM1 in stabilizing pol $\eta$ in the nucleus, we hypothesized that mislocalization of NPM1 to the cytoplasm might cause destabilization of pol $\eta$, similar to the effect that we have observed in cells in which NPM1 was knocked down. To this end, we examined pol $\eta$ levels in OCI/AML2 and OCI/AML3 cell lines derived from AML patients, either without or with the NPM1c + mutation, respectively. As can be seen in Fig. 6a, the amount of poln was much lower in the NPM1c+ AML cell line compared with the NPM1wt AML cell line, whereas the POLH mRNA levels were similar (Fig. 6b). Consistently with the lower poln amount, TLS across a TT CPD was lower (twotailed $t$-test, $P$ value $<0.003)$, and more mutagenic $\left(\chi^{2}\right.$-test, $P$ value $<0.01)$ in the NPM1c + AML cells compared with the NPM1wt AML cells (Fig. 6c). Proteasome inhibition using 
a

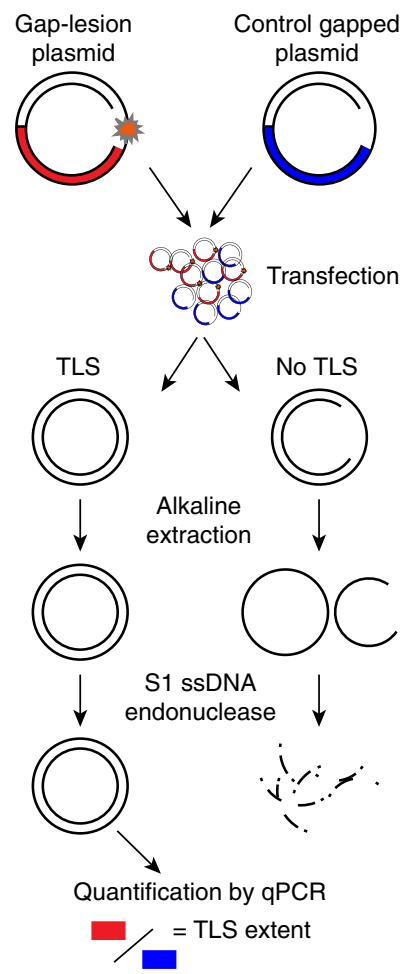

d

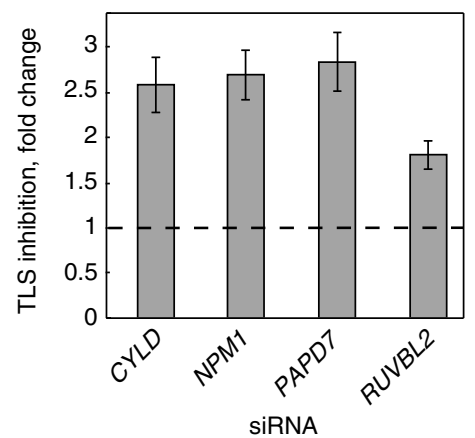

b

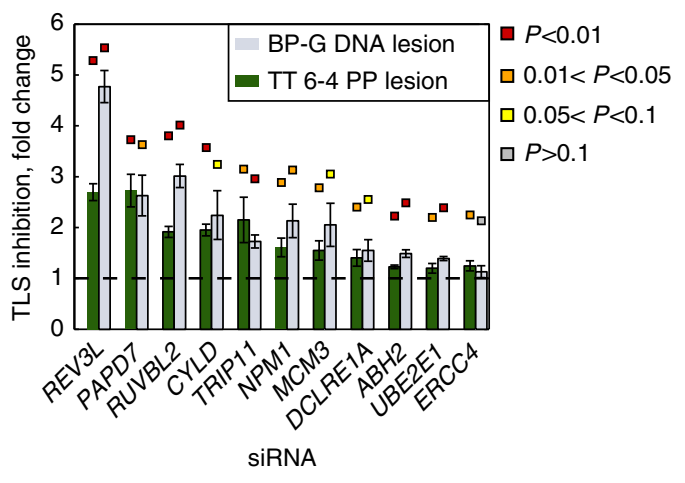

C
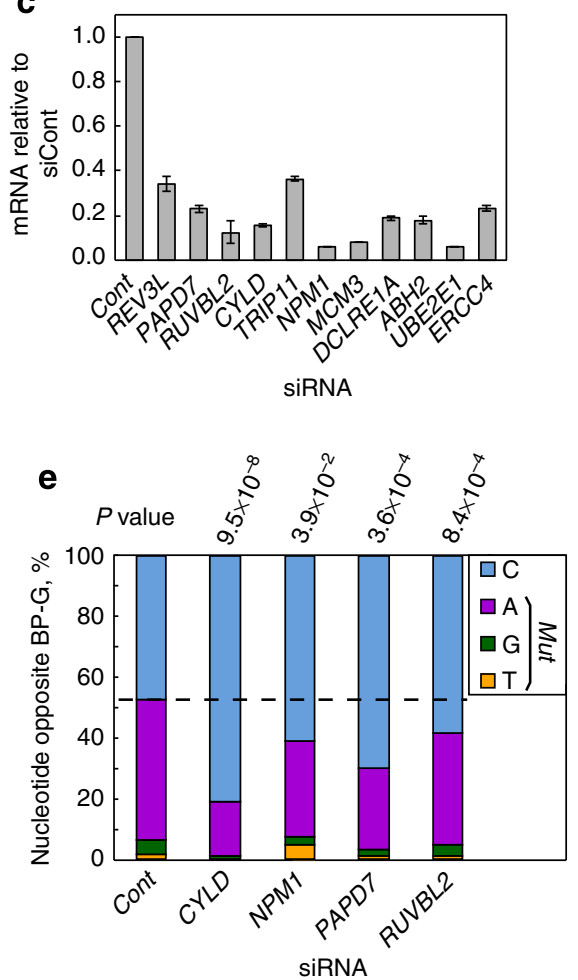

Figure 3 | Validation of TLS candidate genes by qPCR-based and colony-based TLS assays. (a) An outline of the qPCR-based TLS assay. In brief, following TLS in mammalian cells, the plasmids were extracted under alkaline conditions, and subsequently treated with single-stranded-specific endonuclease. This ensures that only filled-in plasmids remain intact. TLS efficiency was calculated as the ratio between the products of qPCRs targeting the filled-in gap-lesion plasmid, and the filled-in control gapped plasmid. (b) qPCR-based TLS assays in MEFs. Ten hit genes were examined for involvement in TLS across the tobacco smoke-induced lesion BP-G and the ultraviolet-induced damage TT 6-4 PP, with Rev3L, encoding the catalytic subunit of DNA polymerase- $\zeta$, serving as a positive control. Data are represented as mean \pm s.e.m. of 4-6 biological replicas. One-sample $t$-test, $P$ values are indicated as a colour code above each column. (c) mRNA knockdown efficiencies by the siRNAs used in $\mathbf{b}$. Values were measured by qPCR and normalized to sample treated with control siRNA (siCont). Mean values \pm s.e.m. of three replicas are presented. (d) Colony-based TLS assay across BP-G in mouse cells. Data are represented as mean \pm s.e.m. of three biological replicas. All the tested genes gave two-tailed $t$-test, $P$ values $<0.02$. (e) Mutagenicity of TLS as observed in the colony-based TLS assays shown in $\mathbf{d}$. Ninety-six colonies were analysed per condition. $\chi^{2} P$ values were calculated relative to control siRNA. ssDNA, single-stranded DNA.

MG132 rescued the level of pol $\eta$ in the NPM1c + AML cell line (Fig. 6d), indicating that pol $\eta$ is subjected to excessive proteasomal degradation, as was the case in cells in which NPM1 was knocked down. To further support the notion that it is the mislocalization of NPM1 in the AML cells that causes the poln deficiency, we treated the cells with Leptomycin B, an inhibitor of nuclear export via exportin 1, previously shown to prevent the exclusion of NPM1 from the nucleus ${ }^{46}$. As can be seen in Supplementary Fig. 8, NPM1 was present mainly in the cytoplasm in NPM1c + AML cells, but not in the NPM1wt AML cells, as expected. Upon addition of Leptomycin B, The NPM1c+
AML cells exhibited nuclear localization of NPM1, similarly to the NPM1wt cells. Examining the level of poln under these conditions revealed that Leptomycin B treatment did not change the amount of poln in NPM1wt AML cells, but caused a significant increase in pol $\eta$ in the NPM1c + AML cells (Fig. 6e), consistent with a role of NPM1 in stabilizing pol $\eta$ in the nucleus. Furthermore, ectopic expression of mCherry-NPM1c + in HEK293 cells that stably express GFP-poln caused a significant twofold decrease in the amount of GFP-pol $\eta$ as indicated by flow cytometry analysis (Fig. 6f,g). This effect was not observed when mCherry-NPM1wt or mCherry alone were expressed (Fig. 6h-k). 
a
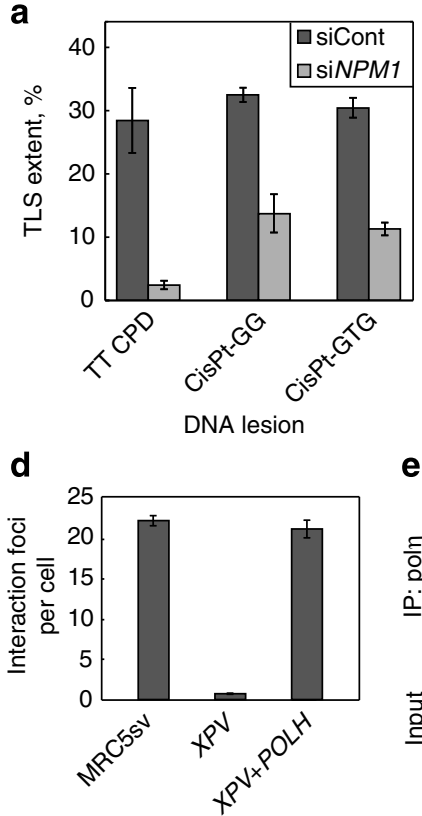

e b

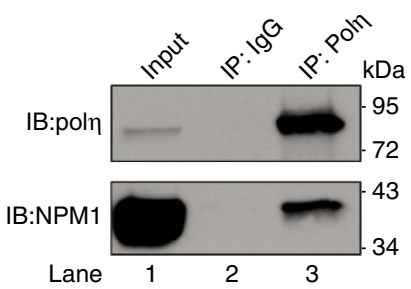

C
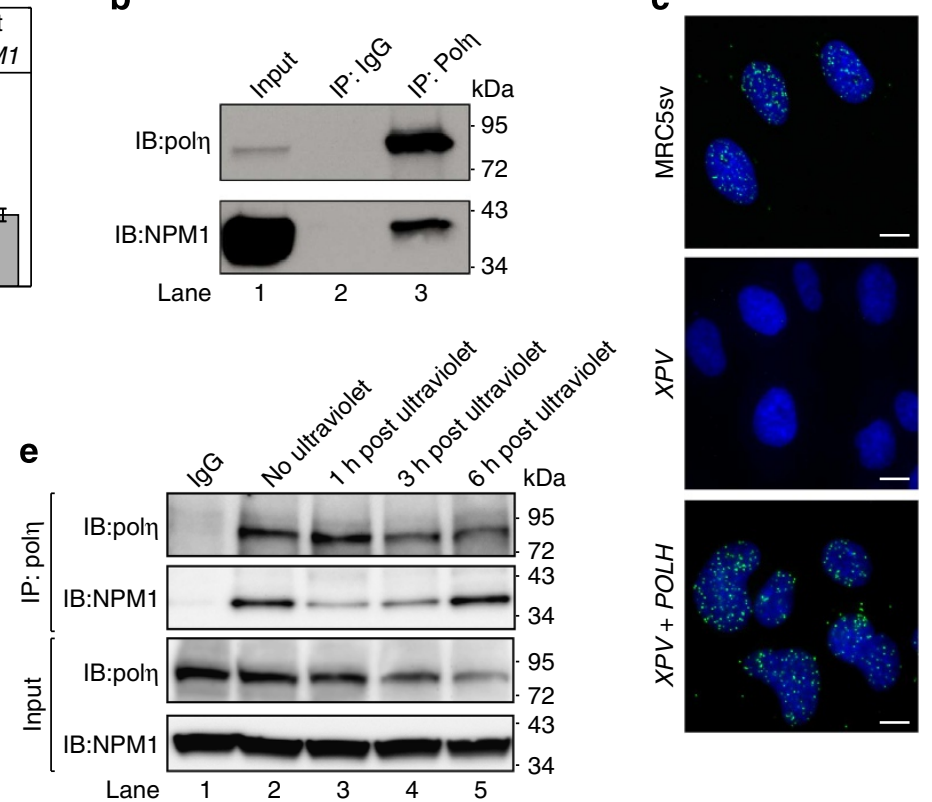

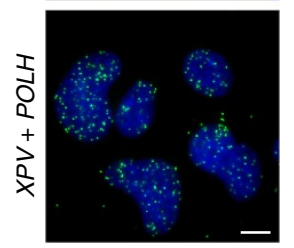

$\mathbf{f}$
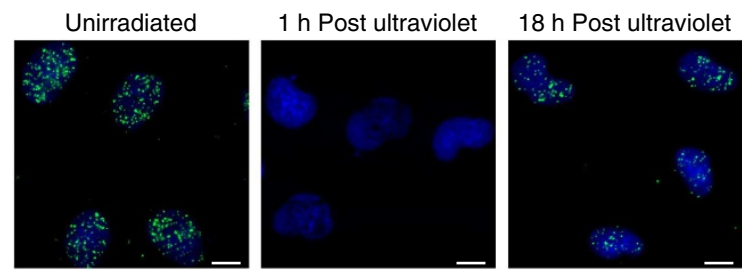

h

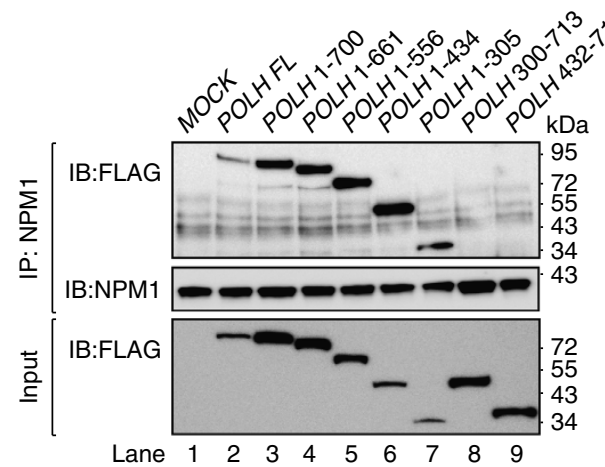

i g

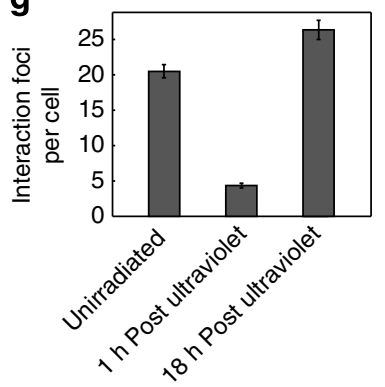

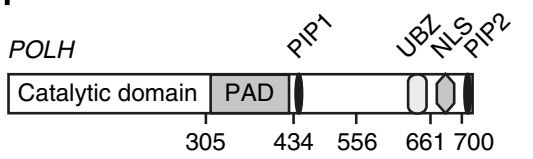

$534 \quad 661700$

Figure 4 | NPM1 interacts with DNA poln and regulates poln-promoted TLS. (a) NPM1 is required for effective TLS across site-specific TT CPD, or the cisplatin-induced site-specific lesions cisPt-GG, and cisPt-GTG in MEFs. Results of gapped plasmid-based, colony-forming TLS assays are presented as the mean \pm s.e.m. of three biological replicas. Two-tailed $t$-test, $P$ values $<0.02$ for all tested lesions. (b) Co-IP of poln and NPM1 in extracts from MRC5sv cells. (c) PLA of pol $\eta$ and NPM1 in MRC5sv cells (upper panel), pol $\eta$-deficient XPV cells (middle panel) and XPV cells complemented with ectopic pol $\eta$ expression (bottom panel). Blue, DAPI (4,6-diamidino-2-phenylindole) staining of the DNA in the nucleus; green, poln-NPM1 interaction detected by the assay. White scale bars correspond to $10 \mu \mathrm{m}$. (d) Quantification of the interacting foci shown in c, based on at least 140 cells of each type. Mean values \pm s.e.m. are presented. (e-g) Poln-NPM1 interaction is transiently lost following ultraviolet irradiation. (e) Co-IP of poln and NPM1 extracted from MRC5sv cells at different time points after ultraviolet irradiation with $10 \mathrm{Jm}^{-2}$. (f) PLA of pol $\eta$ and NPM1 in unirradiated and ultraviolet irradiated cells. Blue, DAPI staining of the DNA in the nucleus; Green, poln-NPM1 interaction. White scale bars correspond to $10 \mu \mathrm{m}$. (g) Quantification of the interacting foci shown in $\mathbf{f}$, based on at least 100 cells of each type. Mean values \pm s.e.m. are presented. (h) NPM1 interacts with the catalytic core of poln. NPM1 was precipitated from HEK293FT cells expressing a serious of FLAG-POLH deletion mutants. Co-precipitation of the FLAG-POLH mutants is shown in the upper panel, whereas the expression of POLH mutants before precipitation is shown in the lower panel. (i) The functional domains of poln. PAD, polymerase-associated domain; PIP1 and PIP2, PCNA-interacting protein domains 1 and 2, respectively; UBZ, ubiquitin-binding zinc finger; NLS, nuclear localization signal. All blots are representative of three independent experiments. IB, immunoblotting; siCont, control siRNA. POLH FL, POLH full length.

Taken together, these results establish a role for NPM1 in the regulation of TLS, and suggest that the prevalent NPM1c+ mutation results in reduced levels of poln, thereby leading to defective TLS.

\section{Discussion}

The multiplicity of TLS DNA polymerases and their low fidelity suggest the existence of a complex regulation to ensure their action at the right place and the right time. The molecular basis of 
a

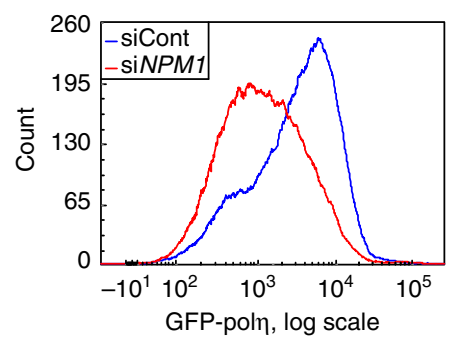

b

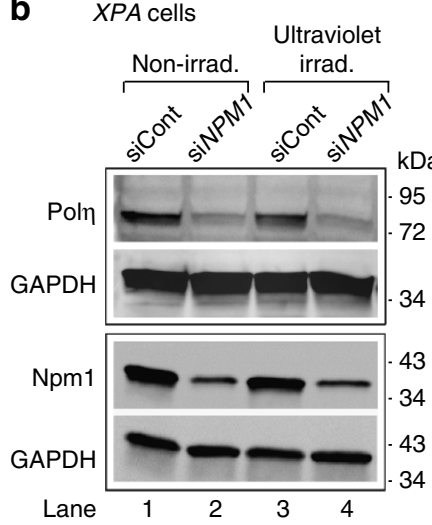

e

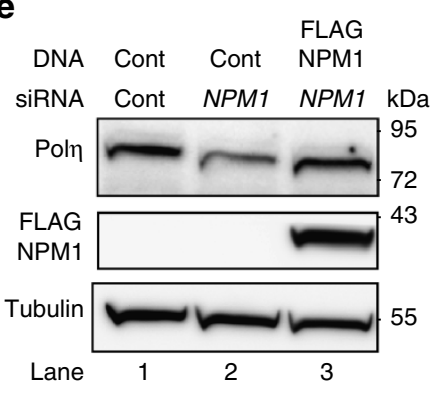

f

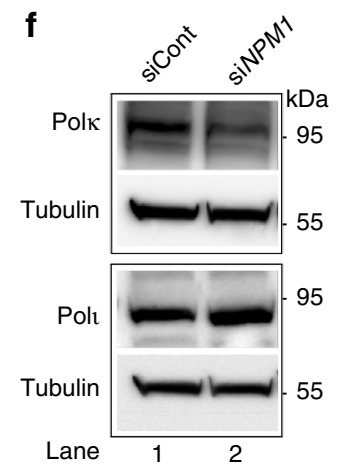

c

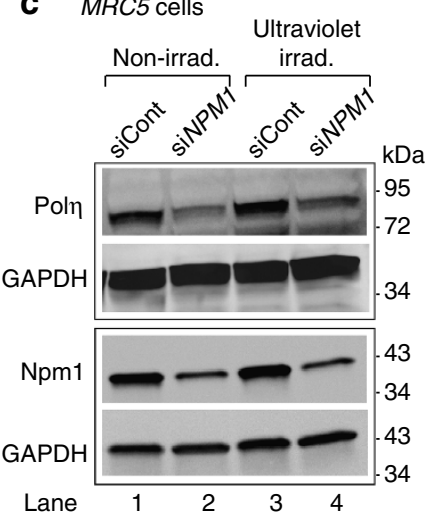

d
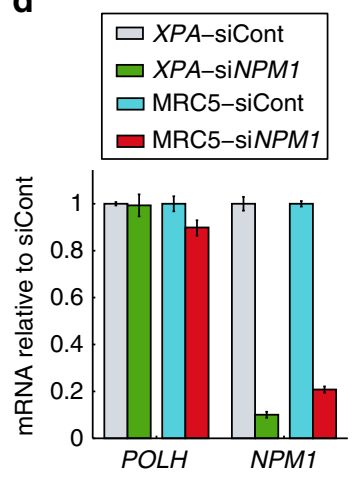

g

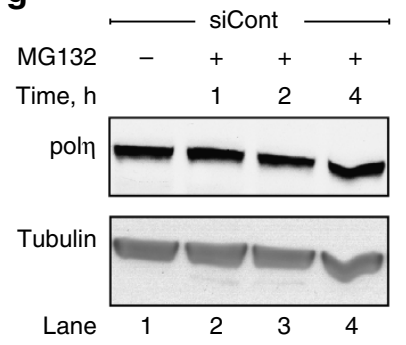

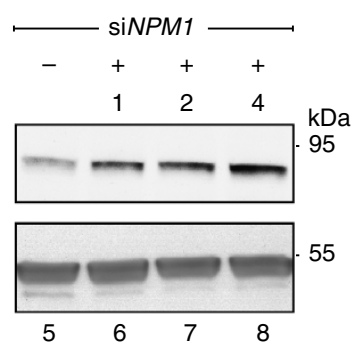

Figure 5 | NPM1 protects DNA poly from proteasomal degradation. (a) MRC5sv cells stably expressing GFP-POLH were treated with siRNA against NPM1 or non- targeting control siRNA (siCont). GFP-poln levels were quantified by flow cytometry. (b,c) Immunoblot of poln extracted from human XPA cells (b) and MRC5sv cells (c) pretreated with siRNA against NPM1 or non-targeting siCont. When indicated, cells were ultraviolet-irradiated $15 \mathrm{~h}$ before extraction with $2 \mathrm{~J} \mathrm{~m}^{-2}$ (b) or $10 \mathrm{Jm}^{-2}$ (c). Upper and lower blots were done with the same extracts. (d) Poln downregulation is not at the transcription level. qPCR of POLH and NPM1 mRNA levels from cells pretreated with siRNA against NPM1, normalized to non-targeting siRNA-treated cells. Mean values \pm s.e.m. of three replicas are presented. (e) Immunoblot of poln extracted from MRC5sv cells pretreated with siRNA against NPM1 and complemented by ectopic expression of an siRNA-resistant NPM1 construct. (f) Polk, but not polı, is also affected by NPM1 knockdown. Immunoblots of polk and polı extracted from MRC5sv cells pretreated with siRNA against NPM1, or non-targeting siCont. (g) Accumulation of poln following proteasomal inhibition. MRC5sv cells were treated with non-targeting siRNA or siRNA against NPM1 and then subjected to MG132 for the indicated time periods in order to inhibit proteasomal activity. Tubulin served as a loading control. All blots are representative of three independent experiments. Irad., irradiated.

the regulation that enables a low and tolerable mutation burden is only partially understood. We reasoned that given its complexity, TLS in mammalian cells is regulated by additional as yet unidentified genes, and searched for those using a two-stage functional siRNA screen. Having two functional assays, one of which was specific for TLS, and a subsequent stringent validation protocol that included analysis by additional TLS assays, was anticipated to reduce the number of false hits. The high selectivity of the screens for TLS genes is demonstrated by the high enrichment of known TLS genes within the hits, in contrast to other DNA repair pathways. The switch to mouse cells for the subsequent $\mathrm{qPCR}$ validation enabled us to gain more validation power by using different siRNA oligos to target the mouse genes, and by demonstrating that the hits are not cell type- or humanspecific. It should be noted that the secondary gapped plasmidbased screen used in this study measures TLS outside the chromosomal context, and hence TLS genes that are associated with chromatin structure or replication fork progression are unlikely to be scored. Such TLS genes might have been detected in the primary ultraviolet-sensitivity screen. The E3 ligase RAD18 that mediates PCNA monoubiquitination was not scored in our screen, although we have previously shown that it is required for effective TLS in the gap-lesion plasmid assay ${ }^{23}$. This might have been caused by inefficient knockdown of the gene. Our screening strategy had led to the identification of 17 new TLS genes, six of which, namely NPM1, SMURF2, UBE2E1, CYLD, OTUB2 and VCPIP1, do not have obvious homologues in E. coli or $S$. cerevisiae. Of the 17 TLS genes, 10 were further examined by a transcription-/translation-independent qPCR version of the TLS assay in mouse cells, and at least four, namely CYLD, NPM1, $P A P D 7$ and RUVBL2, also affected mutation rates across the BPG lesion, further supporting their role in TLS. Most of our hits were detected when screening for TLS across the TT 6-4 PP lesion, with only few exceptions detected in the TT CPD screen, perhaps owing to the robustness of CPD bypass by the highly specialized poln. Virtually, all of our hits seem to have a positive role in TLS, with the exception of USP1, which is a known negative TLS regulator responsible for de-ubiquitination of monoubiquitinated PCNA ${ }^{47}$.

Most of the new TLS hits can be classified into four functional categories, including DNA replication and repair genes, genes affecting proteasomal degradation, chaperons and Golgi-related genes (Table 1). The replication/repair category contains five genes, including the helicase subunit $M C M 3$, which is involved in the formation of replication forks; $A L K B H 2$, which is involved in a direct reversal repair pathway; DCLRE1A, which functions in the repair of DNA crosslinks; ERCC4, which function in HRR as well as in NER; and RUVBL2. Scoring those genes as regulators of 
a

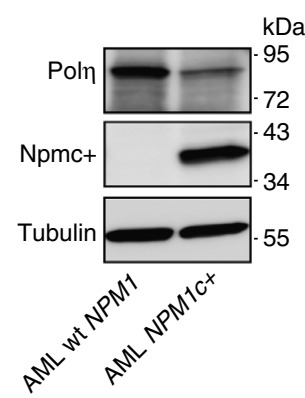

d

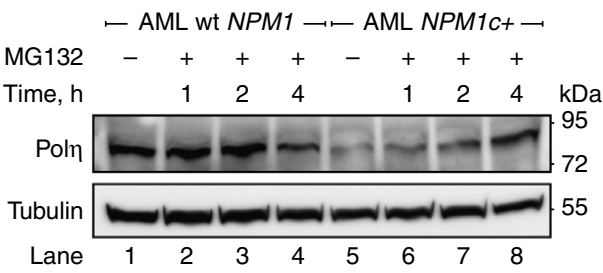

b

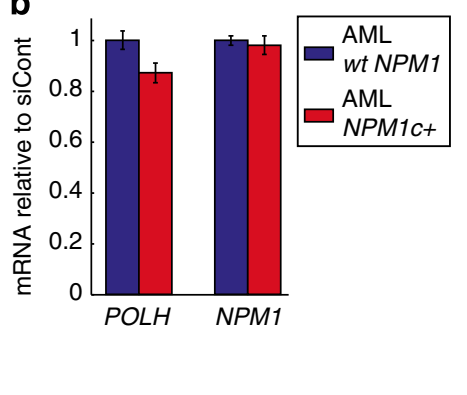

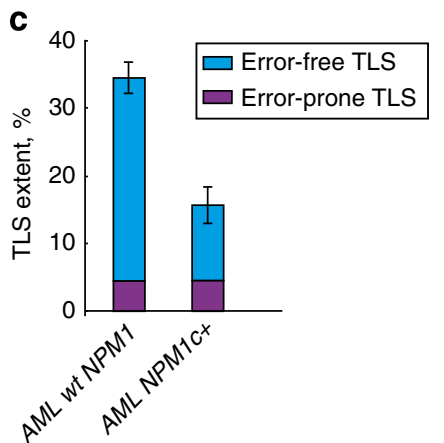

e

e $\quad-$ AML wt NPM1 - $-\mathrm{AML} N P M 1 C+-$

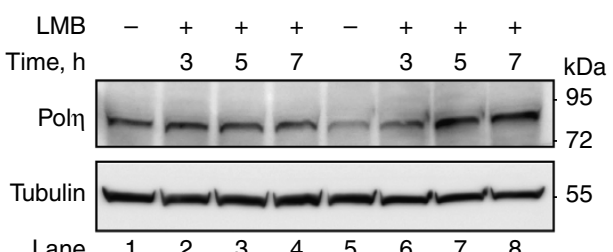

f

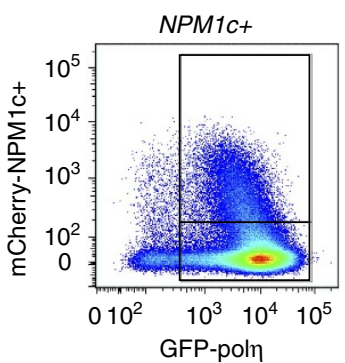

g

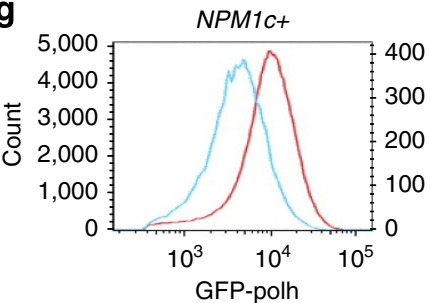

h

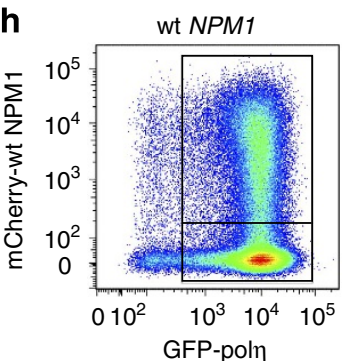

i

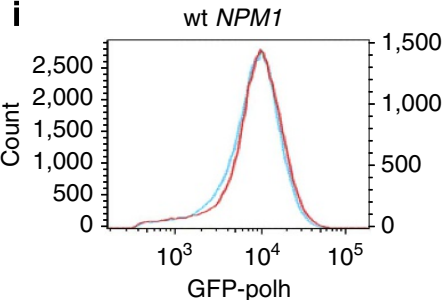

j
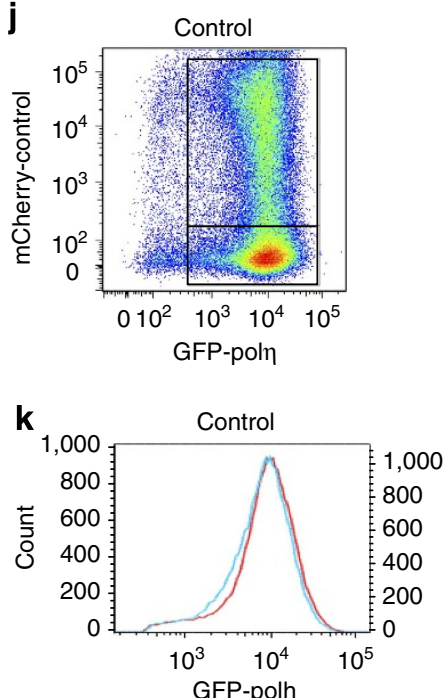

Figure 6 | The NPM1c + mutation causes DNA poln degradation and TLS deficiency. (a) Immunoblot of poln extracted from OCI/AML2 and OCI/AML3 cells expressing wt NPM1 and NPM1c + alleles, respectively. (b) qPCR of POLH and NPM1 mRNA levels from OCl/AML2 and OCl/AML3 cells. Mean values \pm s.e.m. of three replicas are presented. (c) TLS in OCl/AML2 and OCl/AML3 cells. TLS across a site-specific TT CPD using the gapped plasmid-based, colony-forming TLS assay is shown. Mean values \pm s.e.m. of three replicas are presented, two-tailed $t$-test, $P$ value $<0.003$ ). Mutagenicity was addressed by sequencing 96 TLS events per cell line, $\chi^{2}$-test, $P$ value $<0.01$. (d) Proteasomal inhibition rescued Pol $\eta$ deficiency in NPM1c + AML cells. Pol $\eta$ levels in OCl/ AML2 and OCl/AML3 cells pretreated with MG132 for the indicated time to inhibit proteasomal activity. Tubulin served as a loading control. (e) NPM1 nuclear retention rescued Poln deficiency in NPM1c + AML cells. Pol $\eta$ levels in OCl/AML2 and OCI/AML3 cells pretreated with Leptomycin B (LMB) for the indicated time or its solvent as a control. Tubulin served as a loading control. (f-k) Density plots and histograms representing fluorescence-activated cell sorting analysis of HEK293 cells stably expressing GFP-pol $\eta$, and partially transfected with mCherry-tagged NPMc + (f,g), wt NPM1 (h,i) and control mCherry (j,k). Data from mCherry-negative (untransfected) cells in ( $\mathbf{g}, \mathbf{i}, \mathbf{k})$ are in red and correspond to the left $y$ axis, while that from mCherry-positive (transfected) cells are in blue and correspond to the right $y$ axis. All blots are representative of three independent experiments. siCont, control siRNA.

TLS suggests cross talk between DNA replication, repair and damage tolerance. Interestingly, the recruitment of DCLRE1A to sites of damage requires the ubiquitination of PCNA by RAD18 in a similar manner to TLS polymerases ${ }^{48}$. Since TLS polymerases are implicated in crosslink repair, it is tempting to speculate that DCLRE1A participates in coordinating the TLS and crosslink repair pathways, a task in which FAAP20 was recently implicated $^{49}$. RUVBL2 plays an essential part in several chromatin-remodeling complexes including INO80 that regulates transcription by sliding nucleosomes on DNA. Of note, the INO80 complex is also required for removal of histones at sites of damage and was recently suggested to regulate DNAdamage tolerance ${ }^{50,51}$.

The next two categories include four genes involved in protein degradation by the proteasome and two genes encoding chaperons, consistent with the importance of regulating the stability of TLS components. UBE2E1 and UBE2G2 are E2 ubiquitin ligases; SMURF2 is an E3 ubiquitin ligase; and RPN1 forms part of the regulatory subunit of the $26 \mathrm{~S}$ proteasome. The chaperons group includes NPM1, which is discussed below, and DNAJC6, which belongs to the DNAJ/HSP40 family of chaperons, and promotes uncoating of clathrin-coated vesicles. 
Of note, the Caenorhabditis elegans homologue of DNAJC6 (w07a8.3) was previously scored as a hit in a mutagenesis screen, which further supports its involvement in TLS ${ }^{52}$. The category of Golgi-related genes includes TRIP11 and VCPIP1. VCPIP1 is a deubiquitinating enzyme that mediates the reassembly of Golgi stacks after mitosis. Importantly, it also interacts with $V C P / p 97$, which was recently found to regulate $\mathrm{TLS}^{21,22,53}$. There are four additional TLS hits including two de-ubiquitination enzymes, namely OTUB2 and CYLD, the latter being a negative regulator of the NF- $\kappa \mathrm{B}$ pathway; SENP2 that participates in SUMO signalling; and PAPD7 that is an RNA-specific ribonucleotidyl transferase that plays a role in the turnover of aberrant mRNAs ${ }^{54}$. The new TLS genes, which belong to diverse pathways and functions, are likely to be useful for further analysis of TLS in mammalian cells and its interaction with other cellular pathways.

The finding that NPM1 is a regulator of TLS adds an unexpected dimension to the role of this multi-functional protein in preventing genome instability ${ }^{55-57}$, and uncovers a new key player of TLS regulation via proteasomal degradation. By protecting pol $\eta$ from degradation, NPM1 enables TLS to fulfil its function of overcoming replication obstacles, thereby preventing DSB and genome instability ${ }^{58}$. It was previously shown that two E3 ubiquitin ligases promote polyubiquitination of pol $\eta$ and target it for proteasomal degradation: MDM2 (ref. 59) and CRL4(CDT2) (ref. 60). In addition, PIRH2 targets pol $\eta$ to proteasomal degradation via a ubiquitinationindependent pathway ${ }^{61}$. Thus, the cellular levels of pol $\eta$ are tightly regulated. Human HSP90 was reported to facilitate the corrected folding of pol $\eta$ into its active form ${ }^{62}$, and GEI-17 was shown to prevent the polyubiquitination and subsequent degradation of poln by CRL4(CDT2) following genotoxic stress in C. elegans ${ }^{60}$. While GEI-17 is dispensable for pol $\eta$ stability at steady state, NPM1 is critical for maintaining a functional pool of pol $\eta$ in the absence of external DNA damaging agents. Thus, even if GEI-17 activity is conserved in mammals, it does not seem to overlap with the role of NPM1 in protecting pol $\eta$ stability, but rather work subsequently. The transient release of poln from NPM1 following ultraviolet irradiation might enable poln to reach damaged sites in the DNA through its affinity to monoubiquitinated PCNA and preform TLS, however, this dynamics of pol $\eta$ needs further investigation.

It was recently reported that pol $\eta$ is involved in the resistance to araC, a drug routinely used to treat AML, which exerts its therapeutic activity via incorporation into DNA and inhibiting replication ${ }^{63}$. Thus, our results may explain, at least in part, the better prognosis and response to chemotherapy treatment of AML patients carrying the NPM1c + mutation, which causes exclusion of NPM1 from the nucleus, leading to a poln deficiency, and therefore more effective killing of the leukaemic cells by drugs such as araC. Poln was recently implicated in the cellular response to doxorubicin treatment ${ }^{64}$. Although we demonstrated that the NPM1c + OCI/AML3 AML cells carry low levels of poln, they were reported to be more resistant to doxorubicin than OCI/AML2 cells $^{65}$. The sensitivity of the AML cells to doxorubicin is most likely governed by processes other than TLS such as DSB repair and apoptosis, which might also be regulated by NPM1. Such processes can mask the effect of poln, which might be secondary for this drug. The results presented here underscore the critical role of NPM1 in maintaining genome stability, and highlight pol $\eta$ and NPM1, and in particular their interaction, as potential targets for development of drugs for AML therapy.

\section{Methods}

Plasmids construction. pGL4.13 and pGL4.73 reporter plasmids (Promega) served as backbones for the reporter gapped plasmids used in the screen. Restriction-free cloning was performed using PfuUltra II Fusion High-Fidelity DNA Polymerase
(Stratagene). The following cloning steps were performed: (i) Bsal and BstX1 restriction sites were mutated; (ii) the SV40 promoter was replaced with a CMV promoter, taken from pRL-CMV (Promega); (iii) the ColE1 origin of replication was relocated to the region in between the CMV promoter and the reporter gene; and (iv) the AMP resistance cassette was replaced with a chloramphenicol resistance one, and was relocated in the firefly construct to the region in between the CMV promoter and the reporter gene. The mCherry-tagged NPM1 and NPM1c + constructs were produced by fusing the mCherry coding region (Clontech) to the carboxyl terminus of the human NPM1 or NPM1c + coding region. FLAG-tagged poln truncations were produced by introducing the FLAG epitope in the amino terminus of the human $P O L H$ coding region, and deleting the indicated fragments by restriction-free cloning. siRNA-resistant human NPM1 construct was design by introducing silence mutations in the sequence corresponding to the siRNA oligo: $5^{\prime}$ GAGCACCAGUUAUCUUUAA-3'.

Cell cultures. SV40-transformed MRC5sv, XPA (XP12RO), XPV (XP30RO) and $X P V^{P O L H}+$ human fibroblasts were gifts from A.R. Lehmann (University of Sussex, Brighton, UK). C57BL/6 MEFs were gifts from N. de Wind (Ludwig Maximilians University, Munich, Germany). OCI/AML2, OCI/AML3 and HEK293FT cells are commercially available. GFP-poln expressing cells were produced by infection of MRC5sv or HEK293FT cells with GFP-POLH encoding lentiviruses, followed by fluorescence-activated cell sorting (BD FACSAria) for GFP-expressing cells. Human fibroblasts were cultured in MEM, AML lines in MEM- $\alpha$ modification, and MEFs and HEK293FT in DMEM (Gibco). Each medium was supplemented with $2 \mathrm{mM}$ alanyl-glutamine (Biological Industries), $100 \mathrm{units}^{-1}$ of penicillin, $100 \mu \mathrm{g} \mathrm{ml}^{-1}$ of streptomycin (Biological Industries) and $10-15 \%$ fetal bovine serum (Gibco). Throughout the screening steps, cells were maintained at $37^{\circ} \mathrm{C}$ in a $5 \% \mathrm{CO}_{2}$ and $4 \% \mathrm{O}_{2}$ atmosphere.

Ultraviolet-sensitivity screen. Human XPA cells were reverse-transfected in 96 well plates with $25 \mathrm{nM}$ siGenome SMARTpool siRNA libraries (Dharmacon), using Hiperfect transfection reagent (Qiagen). Following incubation of 2 days, cells were washed twice with Hanks' buffer (Sigma) and irradiated in Hanks' buffer at a dose of $1 \mathrm{~J} \mathrm{~m}^{-2}$ ultraviolet $\mathrm{C}$ using a low-pressure mercury lamp (TUV 15W G15T8, Philips). Following irradiation, the cells were maintained under growth conditions for $48 \mathrm{~h}$. Cell viability was determined using CellTiter-Glo (Promega). In order to reduce misinterpretation of toxic effects of certain siRNAs, each sample was normalized using its corresponding non-irradiated sample transfected with the same siRNA. Ultraviolet sensitivity was calculated with respect to control wells.

TLS screen. Human XPA cells were transfected with the siRNA hits from the ultraviolet-sensitivity screen at a concentration of $10 \mathrm{nM}$. The cells were incubated for 2 days, after which they were co-transfected with a mixture of $2.5 \mathrm{ng}$ Fluc gaplesion plasmid and $2.5 \mathrm{ng}$ Rluc control gapped plasmid. Following incubation of $24 \mathrm{~h}$, Fluc and Rluc signals were sequentially measured using the dual-luciferase reporter assay (Promega). TLS extent values were calculated as the ratio between Fluc and Rluc signals in respect to samples transfected with control siRNA, and were corrected for having two different reporters as described in the statistical analysis section. Exclusion of false-positive hits due to off-target effects of the siRNA was achieved by de-convolution of selected siRNA pools and testing each of the four siRNA oligos separately. Gap-lesion plasmids were constructed as described $^{66}$.

Statistical analysis of the primary TLS-specific screen. Viability was measured using luminescence readouts for a set of 1,100 siRNA pools with or without ultraviolet irradiation in three independent biological replicas. This can be represented by: $I_{\mathrm{UV}, i j} ; I_{\text {no UV }, i j}$ where ' $i$ ' stands for the gene index (siRNA pool) and ' $j$ ' for the repeat index. All intensity values are given after $\log _{2}$ transformation. The assay has a 96-well format such that each plate contains nine negative controls of several kinds (non-targeting siRNA, RNA-induced silencing complex (RISC)-free siRNA and siRNA targeting XPA, which is already defective in the cells). Normalization was done by setting the mean intensity of the negative controls in each plate to be equal to the mean intensity of the negative control wells over all the plates. This was done separately for unirradiated plates and ultraviolet-irradiated plates, and the data were corrected accordingly. For each plate, the corresponding ultraviolet effect of the negative control wells is given by:

$$
D_{\text {cont }}=\overline{\left[I_{\text {no UV }, i j}-I_{\mathrm{UV}, i j}\right]}
$$

$\forall_{j}=1,2,3$, where average is over $i \in$ negative control wells. Estimation of the noise level was done using intensity-dependent noise model ${ }^{67}$. This estimation assigns a s.d. value to each siRNA according to the mean intensity value. The approach was used since it provides better statistical power and is justified by the log Gaussian nature of the light intensity measurements. The statistic used for the analysis is:

$$
d_{i j}=I_{\mathrm{no} \mathrm{UV}, i j}-I_{\mathrm{UV}, i j}-D_{\mathrm{cont}}
$$

This represents the ultraviolet effect associated with a specific siRNA in a specific repeat with respect to the control ultraviolet effect. According to the null hypothesis, this statistic is distributed with zero mean and an intensity-dependent 
variance,

$$
\mathrm{H}_{0}: d_{i j} \sim N\left(0, \sigma^{2}\left(I_{\mathrm{no} \mathrm{UV}, i j}\right)+\sigma^{2}\left(I_{\mathrm{UV}, i j}\right)\right)
$$

A $P$ value was calculated based on this null hypothesis for each siRNA in each repeat. The three $P$ values calculated for each siRNA were then combined using a Fisher meta $P$ value to get a single $P$ value per siRNA pool. We call a gene 'ultraviolet sensitive' if two conditions are satisfied: $8 \%$ FDR, and a median fold change (FC) of either FC $>1.5$ or FC $<0.8$; these values, which are represented by the red dashed vertical lines on Fig. 1b, were set according to the distribution of the negative control siRNAs.

Statistical analysis of the secondary TLS-specific screen. The TT 6-4 PP screen, TT CPD screen and the NL correction assay where measured each in four independent biological repeats. Thus for each lesion, each siRNA (i) in each replica (j), we have the measured values: $F_{j}^{i} ; R_{j}^{i} ; F_{\mathrm{NL}, j}^{i} ; R_{\mathrm{NL}, j}^{i} ; j=1,2,3,4$, where ' $F$ stands for Fluc intensity and ' $R$ ' for Rluc intensity. Each measured value is compared with a similar experiment with the negative control siRNAs discussed above.

A natural statistic then is (the bar represents average over the four replicas):

$$
T_{i}=\overline{R^{i}}-\overline{F^{i}}-\left(\overline{R^{\text {control }}}-\overline{F^{\text {control }}}\right)-\left[\overline{R_{\mathrm{NL}}^{i}}-\overline{F_{\mathrm{NL}}^{i}}\left(\overline{R_{\mathrm{NL}}^{\text {control }}}-\overline{F_{\mathrm{NL}}^{\text {control }}}\right)\right]
$$

A difficulty arises when analysing this data, namely, the two numbers $F_{j}^{i}$ and $R_{j}^{i}$, measured from the same well, are strongly dependent in terms of transfection efficiency, which is not uniform between repeats and between wells in the same plate. The way we overcome this problem is by a normalization that keeps the difference in each well as was measured originally. This was done by setting the Rluc values to be the same for all replicates and the Fluc values to fit the differences.

$$
F_{j}^{i, \text { new }}=\overline{R^{i}}+d_{j}^{i} ; d_{j}^{i}=F_{j}^{i}-R_{j}^{i} ; R_{j}^{i, \text { new }}=\overline{R^{i}}
$$

Under this normalization:

$$
\operatorname{Var}\left[F_{j}^{i, \text { new }}\right]=\operatorname{Var}\left[d_{j}^{i}\right]
$$

and the intensity distribution does not change. All three assays (TT 6-4 PP screen, TT CPD screen and NL correction) were normalized by this method. When using the new values, the variance of the statistic $T_{i}$ defined above becomes:

$$
\operatorname{Var}\left[T_{i}\right]=\operatorname{Var}\left[\overline{F^{i}}\right]-\operatorname{Var}\left[\overline{F_{\mathrm{NL}}^{i}}\right]
$$

where the values $\operatorname{Var}\left[\overline{F^{i}}\right]$ are taken from intensity-dependent noise estimation, as was done for the ultraviolete-sensitivity screen. Thus, formulating the null hypothesis as:

$$
\mathrm{H}_{0}: T_{i} \sim N\left(0, \operatorname{Var}\left[F^{i}\right]+\operatorname{Var}\left[F_{\mathrm{NL}}^{i}\right]\right)
$$

We chose a threshold that combines $10 \%$ FDR and a median FC of either FC $>1.5$ or FC $<0.7$ (represented by the red dashed vertical lines on Fig. 1f,g), which was set according to the distribution of the negative control siRNAs.

qPCR-based and colony-based TLS assays. MEFs of wt origin were reversetransfected with $10 \mathrm{nM}$ siRNA. Following incubation for 2 days, the cells were cotransfected with the gap-lesion plasmid and the control gapped plasmid that had been used in the TLS screen. Cells were maintained for $12 \mathrm{~h}$, after which their plasmid content was extracted under alkaline conditions, in which only covalently closed plasmids remained non-denatured. In the qPCR-based TLS assay, remnants of nonfilled gapped plasmids were digested by the single-stranded DNA-specific S1-endonuclease (1.6 units $\mathrm{Ll}^{-1}, 30 \mathrm{~min}$ incubation). Reactions were terminated by adding $33 \mathrm{mM}$ EDTA, followed by heat inactivation and purification by a Wizard SV PCR clean-up kit (Promega). TLS analysis was performed by qPCRs using a SYBR Green reagent (KAPA Biosystems). Discrimination between the filled-in gap-lesion plasmid and the control gapped plasmid was achieved based on two primer sets; one targeted the Fluc gene and the other the Rluc gene. The ratio between the two qPCR products, normalized to samples treated with control siRNA, served to assess the extent of TLS. In the colony-based TLS assay, the extracted plasmids were transformed into a TLSdefective $E$. coli recA strain, which was plated in parallel on Luria broth-kanamycin (for repaired gap-lesion plasmids) and Luria broth-chloramphenicol (for repaired control plasmids). The ratio of kanamycin/chloramphenicol resistant $E$. coli colonies represents the efficiency of TLS in the mammalian cells, and sequence analysis of plasmids extracted from individual colonies provides DNA sequence alterations during TLS. The siRNA sequences are listed in Supplementary Table 3.

qPCR for mRNA evaluation. Total RNA was extracted from the cells $48-72 \mathrm{~h}$ after transfection with siRNA, using the Perfect-Pure RNA cultured cells kit (5-PRIME). cDNA was produced using High capacity cDNA reverse transcription kit (Applied Biosystems). A quantity of $5 \mathrm{ng}$ cDNA was taken for qPCR analysis using SYBR Green reagent (KAPA Biosystems). The data was normalized according to measurements of the mouse HPRT and RLP19 genes, or the human GAPDH and HPRT genes. The primers used for the qPCRs are listed in Supplementary Table 4.

Immunoblot analysis. When indicated, cells were transfected with $15 \mathrm{mM}$ siRNA (Dharmacon) using Hiperfect transfection reagent (Qiagen). Cells were maintained under growth conditions for 3 days, after which they were lysed using RIPA buffer supplemented with protease inhibitor cocktail and phenylmethanesulfonyl fluoride (PMSF) (Sigma). For PCNA-ubiquitination measurements, Triton-soluble fractions were extracted by incubating the cells for $5 \mathrm{~min}$ at $4{ }^{\circ} \mathrm{C}$ in buffer A $(100 \mathrm{mM}$ $\mathrm{NaCl}, 300 \mathrm{mM}$ sucrose, $3 \mathrm{mM} \mathrm{MgCl} 2,10 \mathrm{mM}$ PIPES (pH 6.8), $1 \mathrm{mM} \mathrm{EGTA}$ and $0.2 \%$ Triton X-100). Following removal of the buffer (Triton-soluble fraction), cells were rinsed once with cold PBS, and the adhering cellular material (Triton-insoluble fraction) was collected by scrapping and was extracted by incubating at $37^{\circ} \mathrm{C}$ for $45 \mathrm{~min}$ in buffer B (same as buffer A but containing $50 \mathrm{mM} \mathrm{NaCl}$ and no Triton $\mathrm{X}-100)$ supplemented with 60 units DNaseI (Sigma). Protein concentration was determined using the BCA protein assay (Thermo Scientific). Protein extracts were fractionated by SDS-polyacrylamide gel electrophoresis, after which they were transferred to a polyvinylidene difluoride (PVDF) membrane and probed with antibodies specific for poln (B-7, Santa Cruz Biotechnology, diluted 1:500), polı (60,668, Novus, diluted 1:1,000), polк (ab57070, Abcam, diluted 1:1,000), Npm1 (FC82291, Sigma, diluted 1:20,000), FLAG (M2, Sigma, diluted 1:1,000), APE1 (AB194, Abcam, diluted 1:2,000), and tubulin (DM1A, Sigma, diluted 1:20,000). Proteins were visualized using SuperSignal West Pico Chemiluminescent substrate (Pierce). Quantification was done by ImageJ software. Uncropped blots are presented in Supplementary Fig. 9.

Co-immunoprecipitation. Cells were rinsed twice in cold PBS and lysed in Triton lysis buffer $(20 \mathrm{mM}$ Tris-Cl, pH 7.5, $150 \mathrm{mM} \mathrm{NaCl}, 0.5 \mathrm{mM}$ EDTA, $10 \%$ glycerol and $1 \%$ Triton X-100) supplemented with protease and phosphatase inhibitors (Sigma) for $1 \mathrm{~h}$ at $4{ }^{\circ} \mathrm{C}$. Samples were pre-treated with protein A/G PLUS agarose beads (Santa Cruz Biotechnology), and then incubated with $2 \mu \mathrm{g}$ rabbit anti poln (H300, Santa Cruz Biotechnology), $2 \mu \mathrm{g}$ rabbit IgG (Santa Cruz Biotechnology) or $2 \mu \mathrm{g}$ mouse anti FLAG (M2, Sigma) for $2 \mathrm{~h}$, followed by adding protein A/G PLUS agarose beads or Protein A/G MagBeads (Genscripts) for additional $1 \mathrm{~h}$. Beads were washed three times with lysis buffer, and two additional times with lysis buffer that contained also $250 \mathrm{mM} \mathrm{NaCl}$. Beads were boiled in SDS buffer, and were taken for immunoblot analysis.

Immunostaining and PLA. The PLA assay was preformed according to the manufacturer's instructions (OLINK Bioscience). In brief, cells were plated on $12 \mathrm{~mm}$ coverslips pre-coated with $10 \mu \mathrm{g} \mathrm{ml}^{-1}$ fibronectin (Sigma) and fixed with $3 \%$ paraformaldehyde. When indicated, cells were ultraviolet irradiated with $10 \mathrm{~J} \mathrm{~m}^{-2}$ at time points before fixation. Permeabilization was done with $0.1 \%$ Triton X-100. Coverslips were blocked and then probed with rabbit anti poln (AB17725, Abcam, diluted 1:500) and mouse anti-NPM1 (FC82291, Sigma, diluted 1:500). Anti-rabbitplus and anti-mouse-minus PLA probes were added, ligated and amplified according to the manufacturer's instructions. Immunostaining of NPM1 was done using antiNPM1 (FC82291, Sigma, 1:500). Images were captured with Zeiss Axio-Observer Z1 microscope and analysed with CellProfiller software ${ }^{68}$.

Proteasome or nuclear export inhibition. Proteasomal activity was inhibited by treating MRC5sv or AML cells with $10 \mu \mathrm{M}$ MG132 (Sigma) for $1-4 \mathrm{~h}$. NPM1c+ nuclear export was inhibited by treating AML cells with $20 \mathrm{nM}$ Leptomycin B (Santa Cruz Biotechnology) for 3-7 h.

\section{References}

1. Friedberg, E. C. et al. DNA Repair and Mutagenesis (ASM Press, 2006).

2. Lehmann, A. R. \& Fuchs, R. P. Gaps and forks in DNA replication: rediscovering old models. DNA Repair 5, 1595-1498 (2006).

3. Friedberg, E. C. Suffering in silence: the tolerance of DNA damage. Nat. Rev. Mol. Cell. Biol. 6, 943-953 (2005).

4. Prakash, S., Johnson, R. E. \& Prakash, L. Eukaryotic translesion synthesis DNA polymerases: specificity of structure and function. Annu. Rev. Biochem. 74, 317-353 (2005).

5. Livneh, Z., Ziv, O. \& Shachar, S. Multiple two-polymerase mechanisms in mammalian translesion DNA synthesis. Cell Cycle 9, 729-735 (2010).

6. Sale, J. E., Lehmann, A. R. \& Woodgate, R. Y-family DNA polymerases and their role in tolerance of cellular DNA damage. Nat. Rev. Mol. Cell Biol. 13, 141-152 (2012).

7. Sutton, M. D. \& Walker, G. C. Managing DNA polymerases: coordinating DNA replication, DNA repair, and DNA recombination. Proc. Natl Acad. Sci. USA 98, 8342-8349 (2001).

8. Izhar, L., Ziv, O., Cohen, I. S., Geacintov, N. \& Livneh, Z. Genomic assay reveals tolerance of DNA damage by both translesion DNA synthesis and homology-dependent repair in mammalian cells. Proc. Natl. Acad. Sci. USA 110, E1462-E1469 (2013).

9. Berdichevsky, A., Izhar, L. \& Livneh, Z. Error-free recombinational repair predominates over mutagenic translesion replication in E. coli. Mol. Cell 10, 917-924 (2002).

10. Adar, S., Hendel, A., Geacintov, N. \& Livneh, Z. Repair of gaps opposite lesions by homologous recombination in mammalian cells. Nucleic Acids Res. 37, 5737-5748 (2009). 
11. Blastyak, A., Hajdu, I., Unk, I. \& Haracska, L. Role of double-stranded DNA translocase activity of human HLTF in replication of damaged DNA. Mol. Cell. Biol. 30, 684-693 (2010).

12. Branzei, D. \& Foiani, M. Maintaining genome stability at the replication fork. Nat. Rev. Mol. Cell. Biol. 11, 208-219 (2010).

13. Pages, V., Mazon, G., Naiman, K., Philippin, G. \& Fuchs, R. P. Monitoring bypass of single replication-blocking lesions by damage avoidance in the Escherichia coli chromosome. Nucleic Acids Res. 40, 9036-9043 (2012).

14. Masutani, C. et al. The XPV (xeroderma pigmentosum variant) gene encodes human DNA polymerase eta. Nature 399, 700-704 (1999).

15. Johnson, R. E., Kondratick, C. M., Prakash, S. \& Prakash, L. hRAD30 mutations in the variant form of xeroderma pigmentosum. Science 285, 263-265 (1999).

16. Johnson, R. E., Washington, M. T., Haracska, L., Prakash, S. \& Prakash, L. Eukaryotic polymerases $\mathrm{i}$ and $\mathrm{z}$ act sequentially to bypass DNA lesions. Nature 406, 1015-1019 (2000).

17. Shachar, S. et al. Two-polymerase mechanisms dictate error-free and errorprone translesion DNA synthesis in mammals. EMBO J. 28, 383-393 (2009).

18. Hoege, C., Pfander, B., Moldovan, G. L., Pyrowolakis, G. \& Jentsch, S. RAD6dependent DNA repair is linked to modification of PCNA by ubiquitin and SUMO. Nature 419, 135-141 (2002).

19. Kannouche, P. L., Wing, J. \& Lehmann, A. R. Interaction of human DNA polymerase eta with monoubiquitinated PCNA: a possible mechanism for the polymerase switch in response to DNA damage. Mol. Cell 14, 491-500 (2004).

20. Watanabe, K. et al. Rad18 guides pol eta to replication stalling sites through physical interaction and PCNA monoubiquitination. EMBO J. 23, 3886-3896 (2004).

21. Mosbech, A. et al. DVC1 (C1orf124) is a DNA damage-targeting p97 adaptor that promotes ubiquitin-dependent responses to replication blocks. Nat. Struct. Mol. Biol. 19, 1084-1092 (2012).

22. Davis, E. J. et al. DVC1 (C1orf124) recruits the $\mathrm{p} 97$ protein segregase to sites of DNA damage. Nat. Struct. Mol. Biol. 19, 1093-1100 (2012).

23. Hendel, A. et al. PCNA ubiquitination is important, but not essential for translesion DNA synthesis in mammalian cells. PLoS Genet. 7, e1002262 (2011).

24. Huang, T. T. et al. Regulation of monoubiquitinated PCNA by DUB autocleavage. Nat. Cell Biol. 8, 339-347 (2006).

25. Karras, G. I. \& Jentsch, S. The RAD6 DNA damage tolerance pathway operates uncoupled from the replication fork and is functional beyond S phase. Cell 141, 255-267 (2010).

26. Daigaku, Y., Davies, A. A. \& Ulrich, H. D. Ubiquitin-dependent DNA damage bypass is separable from genome replication. Nature 465, 951-955 (2010).

27. Diamant, N. et al. DNA damage bypass operates in the S and G2 phases of the cell cycle and exhibits differential mutagenicity. Nucleic Acids Res. 40, 170-180 (2012).

28. Gohler, T., Sabbioneda, S., Green, C. M. \& Lehmann, A. R. ATR-mediated phosphorylation of DNA polymerase eta is needed for efficient recovery from UV damage. J. Cell Biol. 192, 219-227 (2011).

29. Yamada, M. et al. ATR-Chk1-APC/CCdh1-dependent stabilization of Cdc7ASK (Dbf4) kinase is required for DNA lesion bypass under replication stress. Genes Dev. 27, 2459-2472 (2013).

30. Mansilla, S. F. et al. UV-triggered p21 degradation facilitates damaged-DNA replication and preserves genomic stability. Nucleic Acids Res. 41, 6942-6951 (2013).

31. Soria, G., Podhajcer, O. \& Gottifredi, V. P21Cip1/WAF1 downregulation is required for efficient PCNA ubiquitination after UV irradiation. Oncogene 25, 2829-2838 (2006).

32. Avkin, S. et al. p53 and p21 regulate error-prone DNA repair to yield a lower mutation load. Mol. Cell 22, 407-413 (2006).

33. Albertella, M. R., Green, C. M., Lehmann, A. R. \& O'Connor, M. J. A role for polymerase eta in the cellular tolerance to cisplatin-induced damage. Cancer Res. 65, 9799-9806 (2005).

34. Doles, J. et al. Suppression of Rev3, the catalytic subunit of Pol\{zeta\}, sensitizes drug-resistant lung tumors to chemotherapy. Proc. Natl Acad. Sci. USA 107, 20786-20791 (2010)

35. Wu, F., Lin, X., Okuda, T. \& Howell, S. B. DNA polymerase zeta regulates cisplatin cytotoxicity, mutagenicity, and the rate of development of cisplatin resistance. Cancer Res. 64, 8029-8035 (2004).

36. Xie, K., Doles, J., Hemann, M. T. \& Walker, G. C. Error-prone translesion synthesis mediates acquired chemoresistance. Proc. Natl Acad. Sci. USA 107, 20792-20797 (2010)

37. Zhao, Y. et al. Structural basis of human DNA polymerase eta-mediated chemoresistance to cisplatin. Proc. Natl Acad. Sci. USA 109, 7269-7274 (2012).

38. Ziv, O., Geacintov, N., Nakajima, S., Yasui, A. \& Livneh, Z. DNA polymerase zeta cooperates with polymerases kappa and iota in translesion DNA synthesis across pyrimidine photodimers in cells from XPV patients. Proc. Natl Acad. Sci. USA 106, 11552-11557 (2009)

39. Avkin, S. et al. Quantitative analysis of translesion DNA synthesis across a benzo $[a]$ pyrene-guanine adduct in mammalian cells. The Role of DNA polymerase k. J. Biol. Chem. 279, 53298-53305 (2004).
40. Falini, B. et al. Cytoplasmic nucleophosmin in acute myelogenous leukemia with a normal karyotype. N. Engl. J. Med. 352, 254-266 (2005).

41. Grisendi, S., Mecucci, C., Falini, B. \& Pandolfi, P. P. Nucleophosmin and cancer. Nat. Rev. Cancer 6, 493-505 (2006)

42. Wu, M. H., Chang, J. H. \& Yung, B. Y. Resistance to UV-induced cell-killing in nucleophosmin/B23 over-expressed NIH 3T3 fibroblasts: enhancement of DNA repair and up-regulation of PCNA in association with nucleophosmin/B23 over-expression. Carcinogenesis 23, 93-100 (2002).

43. Federici, L. \& Falini, B. Nucleophosmin mutations in acute myeloid leukemia: a tale of protein unfolding and mislocalization. Protein Sci. 22, 545-556 (2013)

44. Dohner, K. et al. Mutant nucleophosmin (NPM1) predicts favorable prognosis in younger adults with acute myeloid leukemia and normal cytogenetics: interaction with other gene mutations. Blood 106, 3740-3746 (2005).

45. Schnittger, S. et al. Nucleophosmin gene mutations are predictors of favorable prognosis in acute myelogenous leukemia with a normal karyotype. Blood 106, 3733-3739 (2005).

46. Wang, W., Budhu, A., Forgues, M. \& Wang, X. W. Temporal and spatial control of nucleophosmin by the Ran-Crm1 complex in centrosome duplication. Nat. Cell Biol. 7, 823-830 (2005).

47. Kim, J. M. et al. Inactivation of murine Usp1 results in genomic instability and a Fanconi anemia phenotype. Dev. Cell 16, 314-320 (2009).

48. Yang, K., Moldovan, G. L. \& D’Andrea, A. D. RAD18-dependent recruitment of SNM1A to DNA repair complexes by a ubiquitin-binding zinc finger. J. Biol. Chem. 285, 19085-19091 (2010).

49. Kim, H., Yang, K., Dejsuphong, D. \& D’Andrea, A. D. Regulation of Rev1 by the Fanconi anemia core complex. Nat. Struct. Mol. Biol. 19, 164-170 (2012)

50. Falbo, K. B. et al. Involvement of a chromatin remodeling complex in damage tolerance during DNA replication. Nat. Struct. Mol. Biol. 16, 1167-1172 (2009)

51. Papamichos-Chronakis, M. \& Peterson, C. L. The Ino80 chromatin-remodeling enzyme regulates replisome function and stability. Nat. Struct. Mol. Biol. 15, 338-345 (2008)

52. Pothof, J. et al. Identification of genes that protect the C. elegans genome against mutations by genome-wide RNAi. Genes Dev. 17, $443-448$ (2003).

53. Centore, R. C., Yazinski, S. A., Tse, A. \& Zou, L. Spartan/C1orf124, a reader of PCNA ubiquitylation and a regulator of UV-induced DNA damage response. Mol. Cell 46, 625-635 (2012).

54. Ogami, K., Cho, R. \& Hoshino, S. Molecular cloning and characterization of a novel isoform of the non-canonical poly(A) polymerase PAPD7. Biochem. Biophys. Res. Commun. 432, 135-140 (2013).

55. Grisendi, S. et al. Role of nucleophosmin in embryonic development and tumorigenesis. Nature 437, 147-153 (2005).

56. Kurki, S. et al. Nucleolar protein NPM interacts with HDM2 and protects tumor suppressor protein p53 from HDM2-mediated degradation. Cancer Cell 5, 465-475 (2004).

57. Vascotto, C. et al. Functional regulation of the apurinic/apyrimidinic endonuclease 1 by nucleophosmin: impact on tumor biology. Oncogene 33, 2876-2887 (2013)

58. Xia, X. et al. An IKKalpha-nucleophosmin axis utilizes inflammatory signaling to promote genome integrity. Cell Rep. 5, 1243-1255 (2013).

59. Jung, Y. S., Qian, Y. \& Chen, X. DNA polymerase eta is targeted by Mdm2 for polyubiquitination and proteasomal degradation in response to ultraviolet irradiation. DNA Repair 11, 177-184 (2012).

60. Kim, S. H. \& Michael, W. M. Regulated proteolysis of DNA polymerase eta during the DNA-damage response in C. elegans. Mol. Cell 32, 757-766 (2008).

61. Jung, Y. S., Liu, G. \& Chen, X. Pirh2 E3 ubiquitin ligase targets DNA polymerase eta for $20 \mathrm{~S}$ proteasomal degradation. Mol. Cell. Biol. 30, 1041-1048 (2010).

62. Sekimoto, T. et al. The molecular chaperone Hsp90 regulates accumulation of DNA polymerase eta at replication stalling sites in UV-irradiated cells. Mol. Cell 37, 79-89 (2010).

63. Chen, Y. W., Cleaver, J. E., Hanaoka, F., Chang, C. F. \& Chou, K. M. A novel role of DNA polymerase eta in modulating cellular sensitivity to chemotherapeutic agents. Mol. Cancer Res. 4, 257-265 (2006).

64. Moraes, M. C. et al. Both XPA and DNA polymerase eta are necessary for the repair of doxorubicin-induced DNA lesions. Cancer Lett. 314, 108-118 (2012).

65. Lew, Q. J. et al. NPMc(+) AML cell line shows differential protein expression and lower sensitivity to DNA-damaging and p53-inducing anticancer compounds. Cell Cycle 10, 1978-1987 (2011).

66. Ziv, O., Diamant, N., Shachar, S., Hendel, A. \& Livneh, Z. Quantitative measurement of translesion DNA synthesis in mammalian cells. Methods Mol Biol. 920, 529-542 (2012).

67. Zeisel, A., Amir, A., Kostler, W. J. \& Domany, E. Intensity dependent estimation of noise in microarrays improves detection of differentially expressed genes. BMC Bioinformatics 11, 400 (2010).

68. Kamentsky, L. et al. Improved structure, function and compatibility for CellProfiler: modular high-throughput image analysis software. Bioinformatics 27, 1179-1180 (2011). 


\section{Acknowledgements}

We thank Ghil Jona and Tamar Paz-Elizur (Weizmann Institute of Science, Israel) for their help with the siRNA libraries; Isadora Cohen (Weizmann Institute of Science, Israel) for contributing to the manuscript writing; Alan Lehmann (University of Sussex, Brighton, UK) and Niels de Wind (Leiden University Medical Center, The Netherlands) for providing several cell lines. Z.L. is the incumbent of the Maxwell Ellis Chair for Biomedical Research. E.D. is the incumbent of the Henry J. Leir Professorial Chair. This work was supported by grants from the Flight Attendant Medical Research Institute, Florida, USA; the Israel Science Foundation Grant 684/12; the Leona M. and Harry B. Helmsley Charitable Trust, NY, USA (to Z.L.), the Leir Charitable Foundation (to E.D. and A.Z.), the Clore Israel Foundation (to O.Z.), the Associazione Italiana Ricerca Cancro (AIRC)(IG 2013 n.14595, to B.F.) and by the Deutsche Forschungsgemeinschaft (DFG) grant SFB749 (to T.C.).

\section{Author contributions}

O.Z., A.Z. and Z.L. designed the research; O.Z. and N.M.-N. performed the research; A.Z., O.Z., E.D., R.N. N.B.-C. and Z.L. analysed the data; U.S., N.E.G., T.C., S.S., C.E.C., M.P.M., R.R., and B.F. contributed new reagents/analytic tools; O.Z., A.Z., R.N., N.E.G.,

T.C., S.S., C.E.C., M.P.M., B.F., E.D. and Z.L. wrote the manuscript.

\section{Additional information}

Supplementary Information accompanies this paper at http://www.nature.com/ naturecommunications

Competing financial interests: The authors declare no competing financial interests.

Reprints and permission information is available online at http://npg.nature.com/ reprintsandpermissions/

How to cite this article: Ziv, O. et al. Identification of novel DNA-damage tolerance genes reveals regulation of translesion DNA synthesis by nucleophosmin. Nat. Commun. 5:5437 doi: 10.1038/ncomms6437 (2014).

(c) (i) This work is licensed under a Creative Commons Attribution 4.0 International License. The images or other third party material in this article are included in the article's Creative Commons license, unless indicated otherwise in the credit line; if the material is not included under the Creative Commons license, users will need to obtain permission from the license holder to reproduce the material. To view a copy of this license, visit http://creativecommons.org/licenses/by/4.0/ 